\title{
PETROPHYSICAL PROPERTIES OF THE PECHENGA ROCKS OF DIFFERENT METAMORPHIC FACIES
}

\author{
Feliks F. GORBATSEVICH * and Ol'ga M. TRISHINA
}

Geological Institute of the Kola Science Centre RAS, 184209, Apatity, Murmansk region. St. Fersmana 14, Russia

*Corresponding author's e-mail: gorich@geoksc.apatity.ru

\begin{tabular}{l}
\hline ARTICLE INFO \\
\hline Article history: \\
Received 22 March 2017 \\
Accepted 29 August 2017 \\
Available online 25 September 2017 \\
\hline
\end{tabular}

\section{Keywords:}

Pechenga complex

Rocks, metamorphism

Physical properties

Anisotropy

\begin{abstract}
Using rocks composing the Pechenga complex geologic and petrographic peculiarities, metamorphism and physical properties of rocks from prehnite-pumpellyite to amphibolite facies have been studied. The study showed that the rock densities increase from prehnite-pumpellyite to amphibolite facies (Earth's surface). Rocks of epidote-amphibolite and amphibolite facies (Earth's surface) showed the lowest velocities of compression waves. The values of velocity as well as density under depth conditions tend to increase from prehnite-pumpellyite to amphibolite facies. A new quantitative method - acoustopolariscopy was used to distinguish rocks of different facies of metamorphism. The data obtained (density, velocity characteristics, elastic moduli and Poisson's ratios) reflect the actual properties of the massif rocks and can be used for geophysical investigations and technical calculations in mining.
\end{abstract}

\section{INTRODUCTION}

The Pechenga synclinorial structure covers an area of $2300 \mathrm{~km}^{2}$ and consists of North Pechenga and South Pechenga structural-facies zones separated by the Poritash fault. The zones have different patterns of structure and tectonic deformations (Zagorodny et. al., 1964; Kozlowski, 1987; Hanski et al., 1990). Rocks of the northern structural-formational zone of the Pechenga structure are metamorphosed at the prehnite-pumpellyite to middle stages of the amphibolite facies (Zagorodny et al., 1964; Petrov, 1999). At the present erosional surface metamorphic zones as a whole follow the outlines of the Pechenga structural zone with an increase in the intensity of metamorphism to its periphery. The initial stages of the prefolded metamorphism of Pechenga rocks are fixed in parageneses of the prehnite-pumpellyite zone. Inside the Pechenga synclinal structure, there is an extensive area occupied by the Matert Fm. Rocks of this formation have a common geochemical basis (Zagorodny et al., 1964), but they are found in different metamorphic zones - from prehnitepumpellyite to greenschist, epidote-amphibolite and amphibolite. Prehnite-pumpellyite facies was formed in low PT-conditions of metamorphism. At the greenschist facies the influence of PT-conditions is greater. Amphibolite contains a lot of metamorphic minerals such as biotite and amphibole. In this connection, it is interesting to find out how a varying degree of metamorphic reworking affects the physical properties of rocks. Moreover, specific data of rock physical properties of different metamorphic facies will be useful in technical applications.

\section{GEOLOGY OF THE STUDIED AREAS AND PETROGRAPHIC CHARACTERISTICS OF THE SAMPLES}

Rocks of the Matert Formation are located within a few metamorphic zones ranging from prehnite-pumpellyite in the centre of the structure to amphibolite in the southern and south-western parts. In accordance with this, stone material for the studies was selected from metamorphosed diabases and spatially associated with them bodies of gabbro and gabbro-diabases at several sites located within different metamorphic zones (Fig. 1): prehnitepumpellyite (Soukerjoki site), greenschist (Schuoni and Tuljavr sites), epidote-amphibolite (Valasjoki site), amphibolite (Mt Kuchin-tundra site).

Soukerjoki site. The site is located in the northern part of the Pechenga structure, metavolcanic rocks were observed. They are presented by basaltic rocks: diabases, spherical lavas, lava- and tuff breccias and tuffs, which accounted for a major part of the sequence volume. Predominant among volcanic derivatives are the covers of metadiabases and horizons of globular lavas. Lava- and tuff breccias occur together with them and form rare strata and lenses in the sequence section. There is a $45-50^{\circ}$ dip of crystallization schistosity in the rocks to the south and south-east. Metagabbrodiabases forming bodies of north-east and meridional strike are most common 


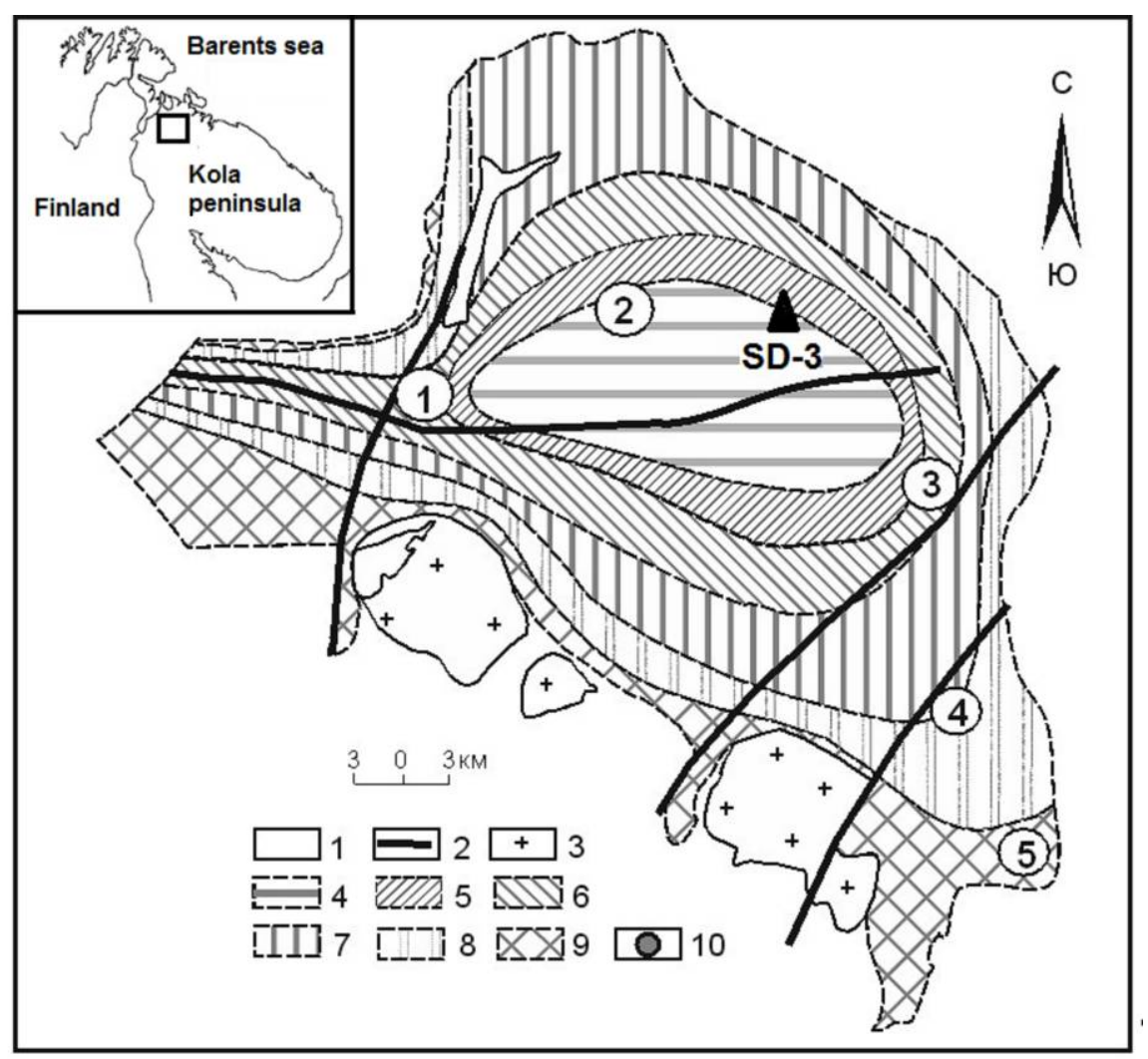

Fig. 1 Map of metamorphic facies of the Pechenga structure (Petrov, 1999) with the sampling sites. 1 - rocks of the Archaean basement, 2 - rock faults, 3 - granitoids, 4-9 - metamorphic zones: 4 prehnite-pumpellyite, 5 - chlorite-actinolite with prehnite, 6 - chlorite-actinolite, 7 - biotite-actinolite, 8 - epidote-hornblende, 9 - amphibolite; 10 - sampling sites (numbers in circles): 1 - Shuoni, 2 - Soukerjoki, 3 - Tuljavr, 4 - Valasjoki, 5 - Mt Kuchin-tundra.

among the intrusive formations. The rocks are metamorphosed at prehnite-pumpellyite facies $(T=$ 220-310 ${ }^{\circ} \mathrm{C}, P=2.5-3.1$ kbar, Fig. 2) and often retain the textures of magmatic rocks, - poikilophitic, intersertal, for globular lavas, - hyalopilitic (Petrov, 1999).

Tuljävr and Shuoni sites. Rocks of greenschist facies have been studied in the area of Lake Tulijavr and middle reaches of the River Shuonijoki located, respectively, in the south-eastern and western parts of the Pechenga structure. In these areas massive and spherical lavas of the Pilgujärvi group intersected by the sheet bodies of gabbro-diabases were examined and tested. In rare cases thin layers of felsic volcanites were observed among metabasalts. The rocks are metamorphosed at greenschist facies at $T=340$ $400{ }^{\circ} \mathrm{C}$ and $P=3-5$ kbar (Petrov, 1999). The textures of metadiabases are granoblastic, nematogranoblastic and in places xenomorphic-granular.

Valasjoki site is located in the south-eastern Pechenga structure. Rocks of basaltic composition are represented by schistose amphibolites metamorphosed at epidote-amphibolite facies at $T=420-470{ }^{\circ} \mathrm{C}$ and $P=4.9-5.4$ kbar (Petrov, 1999). The dip of crystallization foliation in the rocks is western, west-south-western, the dip angles are $45-50^{\circ}$. Amphibolites are crossed by quartz veins with voids, filled with small crystals of quartz. The textures of amphibolites are granoblastic and lepidogranoblastic.

Kuchin-tundra site. In the area of Mt Kuchintundra situated in the far south-east of the North Pechenga zone, there is a fragment of centroclinal structure complicated by the system of steeply dipping rock fractures. The last mentioned separate this structure from the Archaean basement rocks. The main part of Mt Kuchin-tundra is composed of volcanic rocks of the Matert Fm whereas in its southern wall a thin horizon of underlying "black shales" occurs - tufogenic-sedimentary rocks of the Zhdanov Fm enriched in sulphides. Rocks of all underlying formations wedge out as they approach Mt Kuchin-tundra from the north. The rocks are metamorphosed at amphibolite facies by $T=470$ $580{ }^{\circ} \mathrm{C}, P=4.9-5.4$ kbar (Smol'kin, 1992). Schistose amphibolites (metadiabases) are fine-grained, equigranular rocks of granonematoblastic texture. There are signs of cataclasis: shearing accentuated by the orientation of amphibole grains.

The rock species were sampled in the area of the rivers Soukerjoki, Shuoni, Valasjoki, Lake Tuljavr and Mt Kuchin-tundra of the Pechenga structure. These rocks are presented by metamorphic complexes from prehnite-pumpellyite to amphibolite metamorphic facies. The location of the sampling sites 


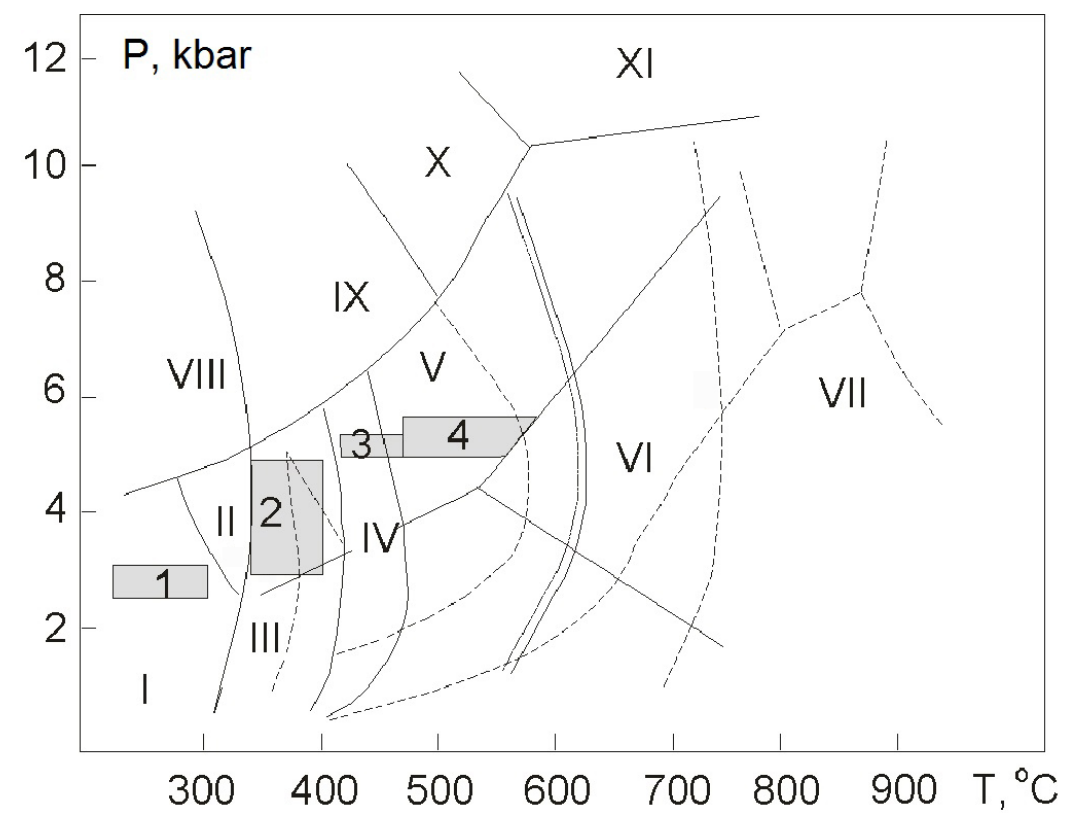

Fig. 2 Location of the sampling sites of rocks metamorphosed at prehnite-pumpellyite to amphibolite facies, Pechenga formation, in the diagram of metamorphic facies by Glebovitsky (1973).

1 - River Soukerjoki (prehnite-pumpellyite); 2 - River Shuoni, Lake Tuljavr (greenschist); 3 - River Valasjoki (epidote-amphibolite); 4 - Kuchin-tundra (amphibolite). Roman numerals - metamorphism facies: I - prehnite-pumpellyite, II - pumpellyite-actinolite, III - greenschist, IV - epidote-amphibolite, V amphibolite, VI - high temperature amphibolite, VII- granulite, VIII - lawsonite-glaucophane-quartz, IX paragonite-zoisite-kyanite, X - kyanite-quartz, XI - kyanite-eclogite.

is shown on the map of the Pechenga formation, Figure 1, and on the diagram of metamorphic facies, Figure 2 (Glebovitsky, 1973).

\section{METHODS OF INVESTIGATION}

Petrographic description of rocks and determination of their mineral composition was performed by the thin sections. For the petrophysical measurements the samples were prepared in the form of a cube with the edge of $2.5-3 \mathrm{~cm}$, Figure 3 . The rock density was determined by the Archimedes method. Compression and shear wave velocities were determined by the acoustopolarization method (Gorbatsevich, 2009) using the device acoustopolariscope (Patent № 1281993, USSR, 1987). The device comprises a radiator and receiver of purely shear linearly polarized ultrasonic waves (Patent No. 785737, USSR, 1980), a goniometer and indicator of the platform rotation angles.

The acoustopolariscope transducers are connected to the ultrasonic flaw detector. The measurements were carried out at the device operating frequency of $1.2 \mathrm{MHz}$. Before the measurements the sample was placed on the rotating platform of the acoustopolariscope. A contact medium wellconducting shear waves was applied to the working surfaces of the radiator and receiver. During the measurements the electric drive rotated the platform within the full rotation angle of $360^{\circ}$ (Kovalevskiy, 2009). The envelope amplitude of the passing wave pulse was fixed on the screen of the ultrasonic device.
At the first stage the measurements are made at parallel polarization vectors (PV) of the source and receiver (VP position). The amplitudes of the waves that have passed through the sample are measured. At the second stage the PVs of the transducers are set at a right angle (the $\mathrm{VC}$ position). The measurement results are VP and VC acoustopolarigrams - circle diagrams of changes in the amplitude of the pulse envelope within the full rotation angle of the rotating platform. The measurements are made on all three pairs of faces of the cubic sample, Figure 3.

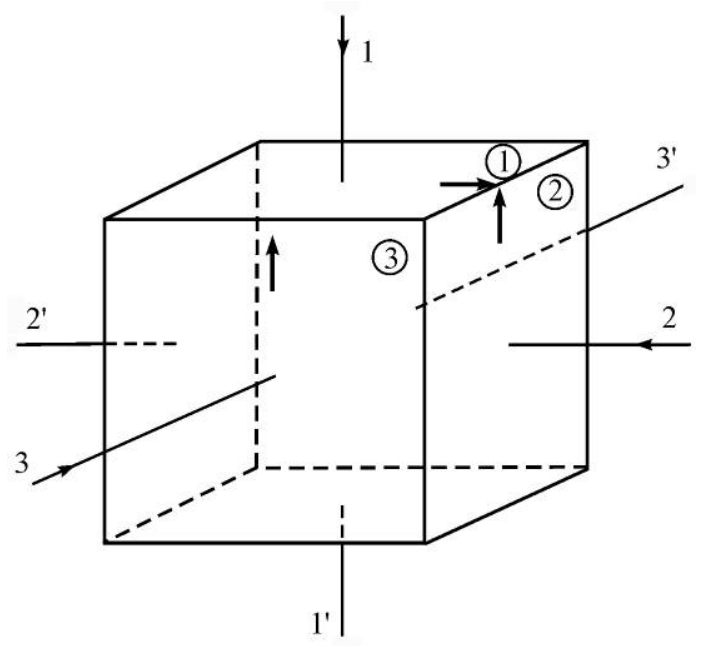

Fig. 3 Sketch of a cubic sample and marking its axes. 
Pleochroism is observed as the light passes through transparent media (Shurkliff, 1962; Berry et al., 1983). It reflects an ability to absorb a beam of polarized light in various ways when rotating its polarization vector with respect to the medium structural elements. In this case, as a rule, the largest absorption of the polarized light is observed when the polarization vector is directed perpendicularly to linearly stretched structural elements of the medium. It is known that rocks, especially metamorphic, often have directed structures (Allison and Palmer, 1980). In this case, by analogy with the optical phenomenon pleochroism, one can observe the phenomenon of LAAA, - the effect of linear acoustic anisotropic absorption during propagation of shear waves (Gorbatsevich, 2009). Depending on the absorption degree the VP acoustopolarigrams acquire the elongated shape.

The acoustopolarigrams obtained at the VC position allow one to determine the number and orientation of the projections of elastic symmetry elements of the anisotropic sample. The orientation of the elastic symmetry elements of the projections on the three faces of the cube were determined from the VC acoustopolarigrams. These projections are the directions, in which the compression and shear wave velocities take extreme values. A more detailed description of the acoustopolariscopy method is given in (Gorbatsevich, 2009).

The measurement results of the compression $\left(V_{\mathrm{P}}\right)$ and shear $\left(V_{\mathrm{S}}\right)$ wave velocities for all the faces of the cubic sample are determined in the form of a quasimatrix (Gorbatsevich et al., 1997),

$V_{\mathrm{ij}}=\begin{array}{lll}V_{11} & V_{12} & V_{13} \\ V_{21} & V_{22} & V_{23} \\ V_{31} & V_{32} & V_{33}\end{array}$,

where $V_{11}, V_{22}, V_{33}$ are compression wave velocities measured in the directions 1-1', 2-2', 3-3'; $V_{12}$ and $V_{13}$ are shear wave velocities measured in the direction 11 ' with PVs in the directions 2-2', 3-3'; $V_{21}, V_{23}-$ in the direction 2-2' with the PVs of the shear wave radiator in the directions 1-1', 3-3 '; $V_{31}, V_{32}$ - in the direction 3-3' with PVs in the directions 1-1', 2-2', respectively. formula

The anisotropy factors were calculated by the

$$
A_{P}=\frac{1}{V_{A V}} \cdot \sqrt{\left(V_{11}-V_{A V}\right)^{2}+\left(V_{22}-V_{A V}\right)^{2}+\left(V_{33}-V_{A V}\right)^{2}},
$$

where $V_{A V}=\left(V_{11}+V_{22}+V_{33}\right) / 3$ is the mean value of compression wave velocity in an anisotropic sample.

To assess the anisotropy degree of the sample from the shear wave velocity the generalized anisotropy factor $B_{S}$ was calculated by the formula:

$$
B_{S}=\sqrt{B_{1}^{2}+B_{2}^{2}+B_{3}^{2}}
$$

where $\quad B_{1}=\frac{2 \cdot\left(V_{12}-V_{13}\right)}{\left(V_{12}+V_{13}\right)} ; \quad B_{2}=\frac{2 \cdot\left(V_{21}-V_{23}\right)}{\left(V_{21}+V_{23}\right)}$;

$$
B_{3}=\frac{2 \cdot\left(V_{31}-V_{32}\right)}{\left(V_{31}+V_{32}\right)}
$$

are factors of shear-wave splitting (Crampin, 1985), defined for the directions 1-1', 2-2', 3-3', respectively. The mean values for the compression wave velocities of the sample were also calculated,

$V_{\mathrm{PR}}=V_{A V}=\left(V_{11}+V_{22}+V_{33}\right) / 3$.

The mean values for the shear wave velocities of the sample were calculated as

$V_{\mathrm{SR}}=\left(V_{12}+V_{13}+V_{21}+V_{23}+V_{31}+V_{32}\right) / 6$.

Because of the weathering and other factors influence rock samples usually collected on the Earth surface have lower density and velocity characteristics $\left(\rho, V_{\mathrm{P}}, V_{\mathrm{s}}\right)$, than those at the depths of $0.1-3 \mathrm{~km}$ and deeper (Handbook 1975; Kern et al., 1990, 2001). The decrease in these characteristics occurs also during stress release (Gorbatsevich, 2003).

This depth range is most interesting for exploration geophysics. Several studies have shown that the differences in the values of $\rho, V_{\mathrm{P}}$ and $V_{\mathrm{S}}$ at great depths are quite near to those determined by their mineral composition (Belikov et al., 1970; Christensen and Mooney, 1995; Rudnick and Fountain, 1995; Kern et al., 2009; Berckhemer et al., 2000; Golovataya et al., 2006). Therefore, the values of the density and velocity of compression and shear waves have been calculated by the rock mineral composition. The calculated values of these velocities considering the specific mineral composition of the rock were taken as initial. The calculations have been done by the formula (Belikov et al., 1970):

$\ln V_{k}=\frac{\Sigma P_{i} \ln \left(V_{i}\right)}{\Sigma P_{i}}$,

where $V_{k}$ is the average calculated velocity in the rock, $V_{i}$ - the average velocity in each mineral, $P_{i}-$ the mineral share in the rock. The densities of the same samples were calculated by a similar formula. The rock mineral composition and corresponding velocity mean values in each particular mineral were taken as initial data (Aleksandrov and Ryzhova, 1961; Belikov et al., 1970; Kern et al., 2009).

On the basis of density and velocity characteristics some technical constants were calculated: elasticity modulus $E$, shear modulus $G$ and Poisson's ratio $v$ by the following formulae:

$E=\left[\rho V_{\mathrm{S}}^{2}\left(3 V_{\mathrm{P}}^{2} / V_{\mathrm{S}}^{2}-4\right)\right] /\left(V_{\mathrm{P}}^{2} / V_{\mathrm{S}}^{2}-1\right)$,

$G=\rho V_{\mathrm{S}}^{2}$,

$v=\left(V_{\mathrm{P}}^{2} / V_{\mathrm{S}}^{2}-2\right) /\left(2 V_{\mathrm{P}}^{2} / V_{\mathrm{S}}^{2}-2\right)$. 
Table 1 Mineral composition and texture of the rock samples in the River Soukeryoki area.

\begin{tabular}{lllll}
\hline Sample № & \multicolumn{5}{c}{ Mineral composition, \% } & \multicolumn{1}{c}{ Texture } & Rock name \\
\hline P-13-31-1 & Pl-43,3 Chl-16,1 Di-14,1 Qtz-6 Bt-1,2 & Fine-grained, massive & Metadiabase \\
& Ilm-2,8 Mag-4,8 Ap-0,3 Srp-4,1 Cb-4,3 & & \\
\hline P-13-33-1a & Pl-48,3 Di- 33,2 Qtz-0,5 Bi-0,7 Ilm-2,6 & Fine-grained, massive, & Gabbro-diabase \\
& gag-4 Ap-0,3 Srp-9 Cb-1,4 & gabbro-diabase & \\
\hline P-13-34-1 & Pl-44,3 Di-30 Qtz-2,2 Bi-2,8 Ilm-2,8 & Medium-inequigranular, & Gabbro \\
& Mag-6,3 Ap-0,2 Srp-6 Ep-5 Cb-0,4 & massive, gabbroic & \\
\hline P-13-35-1 & Pl-38,4 Di-37,2 Qtz-6,7 Ilm-4,2 Mag- & Fine-grained, massive & Gabbro-diabase \\
& 7,6 Ap-0,5 Srp-3 Cb-0,3 Prh-2,1 & & \\
\hline \multirow{2}{*}{ P-13-36-1 } & Pl-44,1 Di-14 Act-22,6 Bi-0,5 Ilm-4,3 & Fine-grained, massive & Pyroxene picrite \\
\hline \multirow{2}{*}{ P-13-36-2 } & Mag-6,8 Ap-0,5 Srp-7 Cb-0,2 & & \\
\hline
\end{tabular}

Note. Srp-serpentine, Cb-carbonate. The remaining symbols are as in (Kretz, 1983). Further, in the other tables on mineral composition symbols are also as in (Kretz, 1983).

Table 2 Petrophysical properties of the samples of prehnite-pumpellyite facies (experimental and calculated data).

\begin{tabular}{|c|c|c|c|c|c|c|c|c|c|c|}
\hline Sample № & Rock name & $\begin{array}{c}\text { Matrix of } \\
\text { velocities } V_{\mathrm{ij}} \\
\mathrm{km} / \mathrm{s}\end{array}$ & $\begin{array}{c}A_{\mathrm{P}}, \\
\%\end{array}$ & $\begin{array}{c}B_{\mathrm{S}}, \\
\%\end{array}$ & $\begin{array}{c}\rho_{\mathrm{R}}, \\
\mathrm{g} / \mathrm{cm}^{3}\end{array}$ & $\begin{array}{c}\rho_{\mathrm{C}}, \\
\mathrm{g} / \mathrm{cm}^{3}\end{array}$ & $\begin{array}{c}V_{\mathrm{PR}} \\
\mathrm{km} / \mathrm{s}\end{array}$ & $\begin{array}{c}V_{\mathrm{PC}} \\
\mathrm{km} / \mathrm{s}\end{array}$ & $\begin{array}{c}V_{\mathrm{SR}} \\
\mathrm{km} / \mathrm{s}\end{array}$ & $\begin{array}{r}V_{\mathrm{SC}} \\
\mathrm{km} / \mathrm{s}\end{array}$ \\
\hline P-13-31-1 & Metadiabase & $\begin{array}{lll}6.40 & 3.27 & 3.25 \\
3.87 & 6.32 & 4.21 \\
3.60 & 3.61 & 5.93 \\
\end{array}$ & 5.6 & 8.4 & 2.63 & 2.90 & 6.22 & 6.18 & 3.64 & 3.41 \\
\hline P-13-33-1a & $\begin{array}{r}\text { Gabbro- } \\
\text { diabase }\end{array}$ & $\begin{array}{lll}6.76 & 3.63 & 3.57 \\
3.51 & 6.46 & 3.46 \\
3.78 & 3.73 & 6.74 \\
\end{array}$ & 3.5 & 2.5 & 2.99 & 2.89 & 6.65 & 6.15 & 3.61 & 3.28 \\
\hline P-13-34-1 & Gabbro & $\begin{array}{lll}6.25 & 3.78 & 3.60 \\
3.69 & 7.09 & 3.81 \\
3.59 & 3.48 & 6.12 \\
\end{array}$ & 11.5 & 6.6 & 2.73 & 2.97 & 6.49 & 6.27 & 3.66 & 3.37 \\
\hline P-13-35-1 & $\begin{array}{r}\text { Gabbro- } \\
\text { diabase }\end{array}$ & $\begin{array}{lll}7.50 & 3.95 & 4.16 \\
3.33 & 5.75 & 3.24 \\
3.61 & 3.66 & 6.36\end{array}$ & 18.9 & 6.0 & 2.98 & 3.01 & 6.54 & 6.23 & 3.66 & 3.37 \\
\hline P-13-36-1 & $\begin{array}{c}\text { Pyroxene } \\
\text { picrite }\end{array}$ & $\begin{array}{lll}6.25 & 3.61 & 3.47 \\
3.44 & 6.57 & 3.49 \\
3.51 & 3.53 & 6.53 \\
\end{array}$ & 3.8 & 4.2 & 3.03 & 2.99 & 6.45 & 6.44 & 3.51 & 3.49 \\
\hline P-13-36-2 & Metagabbro & $\begin{array}{lll}5.38 & 2.89 & 2.86 \\
2.81 & 5.95 & 2.80 \\
3.11 & 3.45 & 5.43 \\
\end{array}$ & 8.0 & 10.3 & 2.59 & 2.99 & 5.59 & 6.44 & 2.99 & 3.49 \\
\hline & AVERAGE & & $\begin{array}{r}8.6 \\
\pm 5.4 \\
\end{array}$ & $\begin{array}{r}6.3 \\
\pm 2.6 \\
\end{array}$ & $\begin{array}{r}2.83 \\
\pm 0.20 \\
\end{array}$ & $\begin{array}{r}2.96 \\
\pm 0.05 \\
\end{array}$ & $\begin{array}{r}6.32 \\
\pm 0.39 \\
\end{array}$ & $\begin{array}{r}6.29 \\
\pm 0.13 \\
\end{array}$ & $\begin{array}{r}3.51 \\
\pm 0.16 \\
\end{array}$ & $\begin{array}{r}3.40 \\
\pm 0.08 \\
\end{array}$ \\
\hline
\end{tabular}

For determining $E, G$ and $v$ under the Earth's surface conditions experimental $\rho_{\mathrm{R}}, V_{\mathrm{PR}}, V_{\mathrm{SR}}$. were used, under the deep conditions, - calculated $\rho_{\mathrm{C}}, V_{\mathrm{PC}}$, $V_{\text {SC }}$ were used (Tables $2,4,6,8$ ). The petrographic description of the rocks, values of density, compression and shear wave velocities, elastic moduli, Poisson's ratios for the surface and deep conditions are given in Tables 1-9.

\section{PREHNITE-PUMPELLYITE FACIES}

In the River Soukerjoki area six samples of gabbro-diabase and gabbro were selected, Figure 1. The photos of the samples' thin sections (crossed nicols) are shown in Figure 4. The petrographic description of rocks and determination of their mineral composition was performed (Table 1). The main rockforming minerals include (in \%) plagioclase (38-48), actinolite (up to 22), diopside (14-37), accessories biotite, often chloritized, ilmenite, orthoclase, apatite, serpentine; quartz and carbonate. According to the petrographic description the rock texture is finegrained, massive, medium-inequigranular and gabbroic.

Samples in the form of a cube, Figure 3, were prepared for the acoustopolariscopy study. The values of density, compression and shear wave velocities, factors of elastic anisotropy etc. were obtained. The petrophysical properties of the samples are shown in Table. 2. It provides the density, velocity quasimatrix $V_{\mathrm{ij}}$ and anisotropy factors $A_{\mathrm{P}}$ and $B_{\mathrm{S}}$. 
Table 3 Mineral composition and texture of the rock samples in the area of Lake Tulijavr and the River Shuonijoki.

\begin{tabular}{|c|c|c|}
\hline $\begin{array}{c}\text { Thin section } \\
\text { № }\end{array}$ & Mineral composition, $\%$ & Rock name \\
\hline P-13-11-1 & $\begin{array}{l}\text { Pl -44,1 Chl-14 Act-22,6 Bi-0,5 Ilm-4,3 Mag- Nematogranoblastic } \\
6,8 \text { Ap-0,5 Srp-7 Cb-0,2 }\end{array}$ & Metadiabase \\
\hline P-13-11-2 & $\begin{array}{llllll}\text { Pl- 42,21 Chl-19,5 } & \text { Act-17 } & \text { Qtz-4 Ilm-4,5 Mag- Lepidonematogranoblastic } \\
\text { 7,8 Ap-0,5 Srp-4,3 } & \text { Cb-0,2 }\end{array}$ & Metadiabase \\
\hline P-13-17-1a & $\begin{array}{l}\text { Pl -47,3 Chl-17 Act-19 Bi -0,8 Ilm-3,3 Mag-6,1 Porphyry } \\
\text { Ap-0,3 Srp-6 Cb-0,2 }\end{array}$ & Metadiabase \\
\hline P-13-20-1 & $\begin{array}{l}\text { Pl -46,5 Chl-27,0 Act-7,6 Bi -1,8 Ilm-3,7 Mag- Ophitic, porphyry } \\
5,8 \text { Ap- } 0,4 \text { Srp-7 Cb-0,2 }\end{array}$ & Gabbro \\
\hline P-13-21-1 & $\begin{array}{l}\text { Pl -24,4 Chl-23,8 Act-21 Bi -3,6 Ilm-6,8 Mag- Nematoblastic cellular } \\
6,5 \text { Ap-0,7 Srp-13 Cb-0,2 }\end{array}$ & Metagabbro \\
\hline P-13-24-1 & $\begin{array}{l}\text { Pl -46 Chl-17,6 Act-12 Bi -1,3 Ilm-3,2 Mag-5,4 f/g nematogranoblastic } \\
\text { Ap-0,4 Srp-7,9 Ep-6 Cb-0,2 }\end{array}$ & Metadiabase \\
\hline P-13-26-1a & $\begin{array}{l}\text { Pl -49,0 Chl-14,2 Act-9,4 Bi -4,6 Ilm-3 Mag-5,3 Ophitic, porphyry } \\
\text { Ap-0,3 Srp-14 Cb-0,2 }\end{array}$ & Metagabbro-diabase \\
\hline P-13-39-1 & $\begin{array}{llll}\text { Pl -41,6 Chl-18,2 Act-7,1 Qtz-11,3 Bi -3,8 Ilm- } & \text { f/g lepido- } \\
\text { 3,9 Mag-5,7 Ap-0,3 Srp-8 } & \text { Cb-0,1 } & & \\
\text { nematogranoblastic }\end{array}$ & Metadiabase \\
\hline P-13-40-1 & $\begin{array}{l}\text { Pl -43,4 Chl-20,2 Act-13 Qtz-7 Bi -3,4 Ilm-2,2 Nematogranoblastic } \\
\text { Mag-5,2 Ap-0,2 Srp-2 Ep-3 Cb-0,4 }\end{array}$ & Metadiabase \\
\hline P-13-41-1 & $\begin{array}{l}\text { Pl -47,2 Chl-31,3 Act-1 Qtz-3,2 Bi -4,1 Ilm-4,2 Nematogranoblastic } \\
\text { Mag-5,2 Ap-0,4 Srp-3 Cb-0,4 }\end{array}$ & Metadiabase \\
\hline P-13-42-1 & $\begin{array}{llllll}\text { Pl -43 Chl-19,4 Act-6 } & \text { Qtz-10,5 Bi-3 Ilm-3,9 Nematogranoblastic } \\
\text { Mag-5,7 } & \text { Ap-0,4 } & \text { Srp-2,2 Ep-5 Cb-0,9 } & & \\
\end{array}$ & Metadiabase \\
\hline
\end{tabular}

Table 4 Petrophysical properties of the samples of greenschist facies (experimental and calculated data).

\begin{tabular}{|c|c|c|c|c|c|c|c|c|c|c|}
\hline Sample № & Rock name & $\begin{array}{c}\text { Velocity matrix, } \\
V_{\mathrm{ij},}, \mathrm{km} / \mathrm{s} \\
\end{array}$ & $\begin{array}{c}A_{\mathrm{P}} \\
\%\end{array}$ & $\begin{array}{c}B_{\mathrm{S}} \\
\%\end{array}$ & $\begin{array}{c}\rho_{\mathrm{R}}, \\
\mathrm{g} / \mathrm{cm}^{3}\end{array}$ & $\begin{array}{c}\rho_{\mathrm{C}}, \\
\mathrm{g} / \mathrm{cm}^{3}\end{array}$ & $\begin{array}{r}V_{\mathrm{PR},} \\
\mathrm{km} / \mathrm{s}\end{array}$ & $\begin{array}{r}V_{\mathrm{PC}}, \\
\mathrm{km} / \mathrm{s}\end{array}$ & $\begin{array}{c}V_{\mathrm{SR}} \\
\mathrm{km} / \mathrm{s}\end{array}$ & $\begin{array}{r}V_{\mathrm{SC}} \\
\mathrm{km} / \mathrm{s}\end{array}$ \\
\hline P-13-11-1 & Metadiabase & $\begin{array}{llll}6.33 & 3.68 & 3.43 \\
4.12 & 7.38 & 4.18 \\
3.28 & 3.38 & 6.11 \\
\end{array}$ & 14.5 & 7.7 & 3.02 & 2.99 & 6.60 & 6.44 & 3.68 & 3.49 \\
\hline P-13-11-2 & Metadiabase & $\begin{array}{lll}6.21 & 3.46 & 3.32 \\
3.68 & 6.76 & 3.85 \\
3.48 & 3.38 & 6.30 \\
\end{array}$ & 6.5 & 6.8 & 2.97 & 3.01 & 6.42 & 6.40 & 3.53 & 3.50 \\
\hline P-13-17-1a & Metadiabase & $\begin{array}{lll}5.33 & 3.18 & 3.03 \\
3.30 & 6.00 & 3.30 \\
3.18 & 3.23 & 5.56 \\
\end{array}$ & 8.6 & 4.8 & 2.91 & 2.95 & 5.63 & 6.40 & 3.20 & 3.46 \\
\hline P-13-20-1 & Gabbro & $\begin{array}{lll}5.72 & 3.11 & 3.16 \\
3.47 & 6.44 & 3.57 \\
3.42 & 3.58 & 6.54 \\
\end{array}$ & 10.1 & 5.6 & 2.78 & 2.95 & 6.23 & 6.29 & 3.38 & 3.37 \\
\hline P-13-21-1 & Metagabbro & $\begin{array}{lll}6.18 & 3.30 & 3.35 \\
3.50 & 6.01 & 3.40 \\
2.90 & 3.02 & 5.17 \\
\end{array}$ & 13.1 & 5.4 & 2.85 & 3.06 & 5.79 & 6.35 & 3.24 & 3.42 \\
\hline P-13-24-1 & Metadiabase & $\begin{array}{lll}6.53 & 3.62 & 3.64 \\
3.40 & 6.20 & 3.48 \\
3.73 & 3.85 & 6.68 \\
\end{array}$ & 5.3 & 3.9 & 2.96 & 2.96 & 6.47 & 6.40 & 3.62 & 3.46 \\
\hline P-13-26-1a & $\begin{array}{l}\text { Metagabbro- } \\
\text { diabase }\end{array}$ & $\begin{array}{lll}6.71 & 3.58 & 3.63 \\
3.50 & 6.21 & 3.51 \\
3.21 & 3.30 & 6.12 \\
\end{array}$ & 7.0 & 3.0 & 2.92 & 2.89 & 6.35 & 6.27 & 3.46 & 3.38 \\
\hline P-13-39-1 & Metadiabase & $\begin{array}{lll}6.58 & 3.75 & 3.58 \\
3.41 & 6.20 & 3.52 \\
3.39 & 3.49 & 6.18 \\
\end{array}$ & 5.1 & 6.3 & 2.95 & 2.92 & 6.32 & 6.26 & 3.52 & 3.46 \\
\hline P-13-40-1 & Metadiabase & $\begin{array}{lll}6.86 & 4.00 & 3.81 \\
3.50 & 6.56 & 3.79 \\
3.41 & 3.46 & 6.13 \\
\end{array}$ & 8.0 & 9.5 & 2.84 & 2.93 & 6.52 & 6.34 & 3.66 & 3.48 \\
\hline P-13-41-1 & Metadiabase & $\begin{array}{lll}6.44 & 2.95 & 3.61 \\
3.75 & 6.50 & 3.80 \\
3.61 & 3.77 & 6.31 \\
\end{array}$ & 2.0 & - & 2.89 & 2.95 & 6.41 & 6.22 & 3.58 & 3.35 \\
\hline P-13-42-1 & Metadiabase & $\begin{array}{lll}7.15 & 3.82 & 4.19 \\
3.54 & 6.28 & 3.64 \\
3.57 & 3.52 & 6.29 \\
\end{array}$ & 10.7 & 9.7 & 2.94 & 2.96 & 6.57 & 6.31 & 3.71 & 3.51 \\
\hline & AVERAGE & & $\begin{array}{r}8.3 \\
\pm 3.5\end{array}$ & $\begin{array}{r}6.3 \\
\pm 2.1 \\
\end{array}$ & $\begin{array}{r}2.92 \\
\pm 0.07\end{array}$ & $\begin{array}{r}2.96 \\
\pm 0.05\end{array}$ & $\begin{array}{r}6.30 \\
\pm 0.31 \\
\end{array}$ & $\begin{array}{r}6.33 \\
\pm 0.07\end{array}$ & $\begin{array}{r}3.51 \\
\pm 0.17\end{array}$ & $\begin{array}{r}3.44 \\
\pm 0.06 \\
\end{array}$ \\
\hline
\end{tabular}


Table 5 The mineral composition and texture of the rock samples in the Valasjoki area.

\begin{tabular}{|c|c|c|c|}
\hline Sample № & Mineral composition, $\%$ & Texture & Rock name \\
\hline $\mathrm{P}-13-45-1$ & 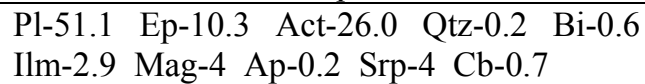 & $\begin{array}{l}\text { Lepidonematogranoblastic, } \\
\text { porphyry }\end{array}$ & Amphibolite \\
\hline P-13-46-1 & $\begin{array}{lrrrr}\text { Pl-40.5 } & \text { Ep-11.9 } & \text { Hbl-19.0 } & \text { Qtz-7 } & \text { Bi-5.2 } \\
\text { Ilm-3.7 } & \text { Mag-9.3 } & \text { Ap-0.4 } & \text { Srp-2 } & \text { Cb-1 }\end{array}$ & $\begin{array}{l}\text { Fine grained, } \\
\text { lepidonematogranoblastic }\end{array}$ & Amphibolite \\
\hline P-13-47-1 & $\begin{array}{lllll}\text { Pl-45.7 } & \text { Ep-11.6 } & \text { Hbl-29.6 } & \text { Qtz-2.2 } & \text { Bi-0.4 } \\
\text { Ilm-2.5 } & \text { Mag-4.7 } & \text { Ap-0.1 } & \text { Srp-3 } & \text { Cb-0.2 }\end{array}$ & Nematogranoblastic & Amphibolite \\
\hline P-13-48-1 & $\begin{array}{llll}\text { Pl-41.8 } & \text { Ep-16.3 } & \text { Hbl-28.0 } & \text { Qtz-10.2 } \\
\text { Ilm-2.7 } & \text { Mag-3.9 } & \text { Ap-0.3 } & \text { Srp-5 Cb-1.1 } \\
\end{array}$ & $\begin{array}{l}\text { Doleritic, xenomorphic- } \\
\text { granular }\end{array}$ & Amphibolite \\
\hline P-13-49-1a & $\begin{array}{l}\text { Pl-43.4 } \\
\text { Ep-12.7 Hbl-25.6 Ol-4.6 Bi -2.1 } \\
\text { Ilm-2.6 } \\
\text { Mag-4 Ap-0.3 Srp-4 Cb-0.7 }\end{array}$ & Lepidonematograno-blastic & Amphibolite \\
\hline P-13-50-1 & $\begin{array}{lllll}\text { Pl-48.8 } & \text { Ep-16.1 } & \text { Hbl-20.0 } & \text { Ol-0.6 } & \text { Bi }-2.5 \\
\text { Ilm-3.2 } & \text { Mag-4.7 } & \text { Ap-0.4 } & \text { Srp-3 } & \text { Cb-0.7 }\end{array}$ & Lepidonematograno-blastic & Amphibolite \\
\hline P-13-51-1 & $\begin{array}{llll}\text { Pl-41.7 } & \text { Ep-20.6 } & \text { Hbl-16.9 } & \text { Qtz-7.6 } \\
\text { Ilm-2.7 } & \text { Mag-5.9 } & \text { Ap-0.4 } \\
\end{array}$ & Nematogranoblastic & Amphibolite \\
\hline
\end{tabular}

Table 6 Petrophysical properties of the samples of epidote-amphibolite facies (experimental and calculated data).

\begin{tabular}{|c|c|c|c|c|c|c|c|c|c|c|}
\hline Sample № & Rock name & $\begin{array}{c}\text { Velocity matrix } \\
V_{\mathrm{ij} .} \mathrm{km} / \mathrm{s}\end{array}$ & $\begin{array}{c}A_{\mathrm{P}} \\
\%\end{array}$ & $\begin{array}{c}B_{\mathrm{S}} \\
\%\end{array}$ & $\begin{array}{c}\rho_{\mathrm{R}} \cdot \\
\mathrm{g} / \mathrm{cm}^{3}\end{array}$ & $\begin{array}{c}\rho_{\mathrm{C}} \cdot \\
\mathrm{g} / \mathrm{cm}^{3}\end{array}$ & $\begin{array}{c}V_{\mathrm{PR}} \\
\mathrm{km} / \mathrm{s}\end{array}$ & $\begin{array}{l}V_{\mathrm{PC}} \\
\mathrm{km} / \mathrm{s}\end{array}$ & $\begin{array}{c}V_{\mathrm{SR}} \\
\mathrm{km} / \mathrm{s}\end{array}$ & $\begin{array}{l}V_{\mathrm{SC}} \\
\mathrm{km} / \mathrm{s}\end{array}$ \\
\hline P-13-46-1 & Amphibolite & $\begin{array}{lll} & \\
5.50 & 3.08 & 3.13 \\
2.92 & 4.98 & 2.97 \\
3.12 & 3.25 & 5.46\end{array}$ & 7.7 & 4.7 & 2.98 & 3.08 & 5.31 & 6.59 & 3.08 & 3.69 \\
\hline P-13-47-1 & Amphibolite & $\begin{array}{lll}4.72 & 3.10 & 2.98 \\
2.89 & 4.48 & 2.72 \\
3.01 & 3.20 & 4.42\end{array}$ & 4.9 & 9.4 & 3.15 & 3.00 & 4.54 & 6.71 & 2.98 & 3.68 \\
\hline P-13-48-1 & Amphibolite & $\begin{array}{lll}4.70 & 3.04 & 3.42 \\
3.21 & 4.72 & 2.93 \\
2.96 & 3.23 & 4.80\end{array}$ & 1.7 & 17.4 & 2.98 & 2.98 & 4.74 & 6.62 & 3.13 & 3.71 \\
\hline P-13-49-1a & Amphibolite & $\begin{array}{lll}6.66 & 3.52 & 3.56 \\
3.53 & 6.70 & 3.58 \\
3.45 & 3.41 & 6.47\end{array}$ & 2.6 & 2.4 & 3.05 & 3.00 & 6.61 & 6.74 & 3.51 & 3.70 \\
\hline P-13-50-1 & Amphibolite & $\begin{array}{lll}4.55 & 3.09 & 2.80 \\
3.42 & 4.72 & 3.33 \\
3.06 & 3.06 & 4.53 \\
\end{array}$ & 3.1 & 10.2 & 2.96 & 3.01 & 4.60 & 6.66 & 3.12 & 3.66 \\
\hline P-13-51-1 & Amphibolite & $\begin{array}{lll}5.01 & 3.35 & 3.12 \\
3.21 & 5.00 & 3.19 \\
3.33 & 3.38 & 4.90 \\
\end{array}$ & 1.7 & 7.5 & 2.92 & 3.04 & 4.97 & 6.62 & 3.26 & 3.73 \\
\hline & AVERAGE & & $\begin{array}{r}3.6 \\
\pm 2.1\end{array}$ & $\begin{array}{r}8.6 \\
\pm 4.8\end{array}$ & $\begin{array}{r}3.01 \\
\pm 0.08\end{array}$ & $\begin{array}{r}3.02 \\
\pm 0.03\end{array}$ & $\begin{array}{r}5.13 \\
\pm 0.78\end{array}$ & $\begin{array}{r}6.66 \\
\pm 0.06\end{array}$ & $\begin{array}{r}3.18 \\
\pm 0.19\end{array}$ & $\begin{array}{r}3.69 \\
\pm 0.02\end{array}$ \\
\hline
\end{tabular}

Table 7 Mineral composition and texture of the rock samples from the Mt Kuchin-tundra area.

\begin{tabular}{|c|c|c|c|}
\hline $\begin{array}{l}\text { Sample } \\
\text { № }\end{array}$ & Mineral composition, $\%$ & Texture & Rock name \\
\hline $\mathrm{v}-1731$ & Hbl-98 Chl-1 Mag-1 & $\begin{array}{l}\text { Medium-coarse-grained, inequigranular, } \\
\text { nematoblastic }\end{array}$ & Amphibolite \\
\hline $\mathrm{v}-1732$ & Hbl-75 Qtz-10 Pl-10 Mag-5 & $\begin{array}{l}\text { Fine-grained, equigranular, } \\
\text { granonematoblastic }\end{array}$ & $\begin{array}{l}\text { Feldspar } \\
\text { amphibolite }\end{array}$ \\
\hline v-1737-1 & Hbl-95 Qtz- 5 & $\begin{array}{l}\text { Medium -grained, equigranular, } \\
\text { nematoblastic fine-spicular and sheaf- } \\
\text { like amphibole }\end{array}$ & Amphibolite \\
\hline v-1734 & Qtz-24 Pl-10 Mag-1 Ttn-5 Hbl-60 & $\begin{array}{l}\text { Medium -grained, equigranular, } \\
\text { granonematoblastic }\end{array}$ & $\begin{array}{l}\text { Feldspar } \\
\text { amphibolite }\end{array}$ \\
\hline $\mathrm{v}-1741-9$ & Pl-34 Di-35 Hbl-26 Ttn-5 & Nematoblastic, relic gabbroic, poikilitic & Metagabbro \\
\hline v-1741-11 & Pl-34 Di-36 Hbl-26 Ttn-4 & Nematoblastic,relic gabbroic, poikilitic & Metagabbro \\
\hline $\mathrm{v}-1741-8$ & Pl-35 Di-35 Hbl-25 Ttn-5 & Nematoblastic,relic gabbroic, poikilitic & Metagabbro \\
\hline $\mathrm{v}-1741-10$ & Pl-33 Di-35 Hbl-27 Ttn-5 & Nematoblastic,relic gabbroic, poikilitic & Metagabbro \\
\hline
\end{tabular}


Table 8 Petrophysical properties of the samples of amphibolite facies (experimental and calculated data).

\begin{tabular}{|c|c|c|c|c|c|c|c|c|c|c|}
\hline Sample № & Rock name & $\begin{array}{c}\text { Velocity matrix } \\
V_{\mathrm{ij}} \cdot \mathrm{km} / \mathrm{s}\end{array}$ & $\begin{array}{c}A_{\mathrm{P}} \\
\%\end{array}$ & $\begin{array}{c}B_{\mathrm{S}} \\
\%\end{array}$ & $\begin{array}{c}\rho_{\mathrm{R}} \cdot \\
\mathrm{g} / \mathrm{cm}^{3}\end{array}$ & $\begin{array}{c}\rho_{\mathrm{C}} \cdot \\
\mathrm{g} / \mathrm{cm}^{3}\end{array}$ & $\begin{array}{r}V_{\mathrm{PR}} \\
\mathrm{km} / \mathrm{s}\end{array}$ & $\begin{array}{l}V_{\mathrm{PC}} \\
\mathrm{km} / \mathrm{s}\end{array}$ & $\begin{array}{r}V_{\mathrm{SR}} \\
\mathrm{km} / \mathrm{s}\end{array}$ & $\begin{array}{r}V_{\mathrm{SC}} \\
\mathrm{km} / \mathrm{s}\end{array}$ \\
\hline \multirow[t]{3}{*}{$\mathrm{v}-1731$} & Amphibolite & $\begin{array}{lll}5.48 & 3.39 & 3.51\end{array}$ & & & & & & & & \\
\hline & & $3.56 \quad 6.35 \quad 3.80$ & 16 & 12 & 3.06 & 3.18 & 6.25 & 7.19 & 3.60 & 3.98 \\
\hline & & $\begin{array}{lll}3.49 & 3.85 & 6.92\end{array}$ & & & & & & & & \\
\hline \multirow[t]{3}{*}{$\mathrm{v}-1732$} & Feldspar & $\begin{array}{lll}5.75 & 3.36 & 3.31\end{array}$ & & & & & & & & \\
\hline & amphibolite & $\begin{array}{lll}3.45 & 6.80 & 3.62\end{array}$ & 12 & 10 & 2.96 & 2.58 & 6.25 & 4.86 & 3.49 & 3.03 \\
\hline & & $3.45 \quad 3.76 \quad 6.21$ & & & & & & & & \\
\hline \multirow[t]{3}{*}{$\mathrm{v}-1734$} & Feldspar & 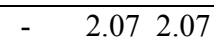 & & & & & & & & \\
\hline & amphibolite & $2.04 \quad 5.462 .91$ & 0 & 35 & 2.87 & 3.06 & 4.98 & 6.77 & 2.51 & 3.94 \\
\hline & & $2.913 .07 \quad 4.51$ & & & & & & & & \\
\hline \multirow[t]{3}{*}{$\mathrm{v}-1737-1$} & Amphibolite & $\begin{array}{lll}6.92 & 3.71 & 3.84\end{array}$ & & & & & & & & \\
\hline & & $\begin{array}{lll}3.66 & 6.37 & 3.72\end{array}$ & 7 & 7 & 2.99 & 3.14 & 6.54 & 7.15 & 3.68 & 4.00 \\
\hline & & $3.66 \quad 3.46 \quad 6.32$ & & & & & & & & \\
\hline \multirow[t]{3}{*}{ v-1741-8 } & Metagabbro & $\begin{array}{llll}5.35 & 3.30 & 3.43\end{array}$ & & & & & & & & \\
\hline & & $3.29 \quad 5.55 \quad 3.35$ & 3 & 5 & 3.29 & 3.02 & 5.48 & 6.88 & 3.37 & 3.81 \\
\hline & & $3.36 \quad 3.47 \quad 5.55$ & & & & & & & & \\
\hline \multirow[t]{3}{*}{ v-1741-9 } & Metagabbro & $4.49 \quad 3.26 \quad 3.17$ & & & & & & & & \\
\hline & & $3.18 \quad 5.75 \quad 3.51$ & 18 & 15 & 3.10 & 3.03 & 5.24 & 6.89 & 3.30 & 3.82 \\
\hline & & $3.16 \quad 3.515 .49$ & & & & & & & & \\
\hline \multirow[t]{3}{*}{ v-1741-10 } & Metagabbro & $\begin{array}{lll}5.91 & 3.37 & 3.64\end{array}$ & & & & & & & & \\
\hline & & $3.52 \quad 5.94 \quad 3.47$ & 0 & 8 & 3.09 & 3.04 & 5.92 & 6.91 & 3.49 & 3.83 \\
\hline & & $3.513 .45 \quad 5.90$ & & & & & & & & \\
\hline \multirow[t]{5}{*}{ v-1741-11 } & Metagabbro & $4.973 .02 \quad 3.15$ & & & & & & & & \\
\hline & & $\begin{array}{lll}3.06 & 5.02 \quad 3.19\end{array}$ & 3 & 8 & 2.95 & 3.01 & 5.06 & 6.89 & 3.14 & 3.82 \\
\hline & & $\begin{array}{llll}3.13 & 3.30 & 5.18\end{array}$ & & & & & & & & \\
\hline & & & 7.4 & 12.5 & 3.04 & 3.04 & 5.72 & 6.69 & 3.32 & 3.78 \\
\hline & AVERAGE & & \pm 7.1 & \pm 9.6 & \pm 0.13 & \pm 0.13 & \pm 0.60 & \pm 0.75 & \pm 0.37 & \pm 0.31 \\
\hline
\end{tabular}

Table 9 Summary table of elastic moduli and Poisson's ratios of rock samples of different facies of metamorphism.

\begin{tabular}{|c|c|c|c|c|c|c|c|}
\hline \multirow[b]{2}{*}{ Metamorphism, site } & \multirow[b]{2}{*}{$\begin{array}{l}\text { Number of- } \\
\text { samples }\end{array}$} & \multicolumn{3}{|c|}{ Earth's surface } & \multicolumn{3}{|c|}{ Deep conditions } \\
\hline & & $\begin{array}{l}E \cdot 10^{-4} \\
\mathrm{MPa}\end{array}$ & $\begin{array}{l}G \cdot 10^{-4} \\
\mathrm{MPa}\end{array}$ & $v$ & $\begin{array}{c}E \cdot 10^{-4} \\
\mathrm{MPa}\end{array}$ & $\begin{array}{l}G \cdot 10^{-4} \\
\mathrm{MPa}\end{array}$ & $v$ \\
\hline $\begin{array}{l}\text { Prehnite-pumpellyite facies, } \\
\text { r. Soukerjoki }\end{array}$ & 6 & $\begin{array}{r}8.97 \\
\pm 1.41 \\
\end{array}$ & $\begin{array}{r}3.51 \\
\pm 0.56 \\
\end{array}$ & $\begin{array}{r}0.277 \\
\pm 0.020 \\
\end{array}$ & $\begin{array}{r}8.86 \\
\pm 0.46 \\
\end{array}$ & $\begin{array}{r}3.43 \\
\pm 0.18 \\
\end{array}$ & $\begin{array}{r}0.293 \\
\pm 0.006 \\
\end{array}$ \\
\hline $\begin{array}{l}\text { Greenschist facies, Lake } \\
\text { Tulijavr, r. Shuonijoki }\end{array}$ & 11 & $\begin{array}{r}9.17 \\
\pm 0.98\end{array}$ & $\begin{array}{r}3.60 \\
\pm 0.40\end{array}$ & $\begin{array}{r}0.275 \\
\pm 0.009\end{array}$ & $\begin{array}{r}9.06 \\
\pm 0.33\end{array}$ & $\begin{array}{r}3.52 \\
\pm 0.20\end{array}$ & $\begin{array}{r}0.290 \\
\pm 0.007\end{array}$ \\
\hline $\begin{array}{l}\text { Epidote-amphibolite facies, } \\
\text { r. Valasjoki }\end{array}$ & 6 & $\begin{array}{r}7.13 \\
\pm 1.36\end{array}$ & $\begin{array}{r}3.05 \\
\pm 0.37\end{array}$ & $\begin{array}{l}0.162 \\
\pm 0.91\end{array}$ & $\begin{array}{r}10.52 \\
\pm .14\end{array}$ & $\begin{array}{r}4.12 \\
\pm 0.07\end{array}$ & $\begin{array}{r}0.278 \\
\pm 0.007\end{array}$ \\
\hline $\begin{array}{l}\text { Amphibolite facies, } \\
\text { Mt. Kuchin-tundra }\end{array}$ & 9 & $\begin{array}{r}8.36 \\
\pm 1.92\end{array}$ & $\begin{array}{r}3.42 \\
\pm 0.69\end{array}$ & $\begin{array}{r}0.213 \\
\pm 0.055\end{array}$ & $\begin{array}{l}11.1 \\
\pm 2.0\end{array}$ & $\begin{array}{r}4.38 \\
\pm 0.80\end{array}$ & $\begin{array}{r}0.263 \\
\pm 0.034\end{array}$ \\
\hline
\end{tabular}

The VP-diagrams (Fig. 5) of the prehnitepumpellyite facies rocks usually have rounded outlines. The acoustopolarigram analysis of the samples of prehnite-pumpellyite facies allows one to assume that these rocks are close to quasi-isotropic. The VC acoustopolarigrams for some samples are minimal and occupy an area close to the coordinate origin, so it is impossible to determine the direction of the symmetry elements. At the $\mathrm{VC}$ position small acoustopolarigrams have been registered except the samples P-13-31-1 (faces 2-2' and 3-3'), P-13-34-1 (faces 1-1' and 3-3') and P-13-36-1 (faces 2-2' and 3$\left.3^{\prime}\right)$.

This is corroborated by the rock texture (Fig. 4). It is massive, fine-grained, mostly without preferential orientation of grains, which also characterizes the samples as quasi-isotropic. The samples P-13-34-1 (faces 2-2 'and 3-3'), P-13-36-1 (faces 2-2', 3-3 ') and $\mathrm{P}-13-36-1$ (faces 2-2 'and 3-3') indicate the presence of the effect of linear acoustic anisotropic absorption (LAAA). The LAAA effect presence in the sample P13-36-1 is caused by a uniform orientation of elongated grains that can be seen in the photographs of thin sections, Figure 4. There is a contact zone on the sample P-13-36-2, which had an impact on the shape of the VP diagram, Figures 4 and 5.

An analysis of the petrophysical properties of the prehnite-pumpellyite facies (Table. 2) shows that changes in the experimentally measured density vary within $2.59-3.03 \mathrm{~g} / \mathrm{cm}^{3}$. The averages for the samples 


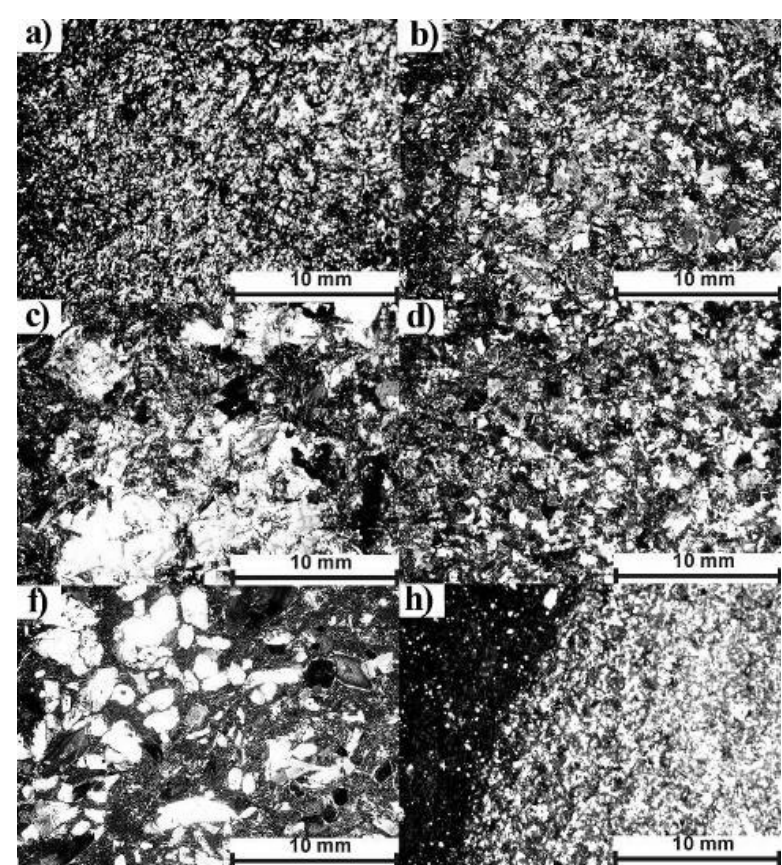

Fig. 4 Photo of thin sections of the samples of prehnite-pumpellyite facies.

a) metadiabase (P-13-31-1); b) gabbro-diabase (P-13-33-1a); c) gabbro (P-13-34-1); d) gabbrodiabase (P-13-35-1); f) pyroxene picrite (P-13-36-1); h) metagabbro (P-13-36-2).

of prehnite-pumpellyite facies for the entire density variety are $2.83 \pm 0.20 \mathrm{~g} / \mathrm{cm}^{3}$. From the review of the quasimatrices of $V_{\mathrm{ij}}$ velocities it follows that each one of the rock velocity characteristics contains a certain (deterministic) and some random (fluctuating) components. The highest and lowest values of the average velocity of compression waves $\left(V_{\mathrm{PR}}\right)$, measured in the samples in the laboratory, vary within $5.59-6.65 \mathrm{~km} / \mathrm{s}$. The shear wave velocities $\left(V_{\mathrm{SR}}\right)$ registered in the samples in the laboratory vary in the range of $2.99-3.66 \mathrm{~km} / \mathrm{s}$. The average velocities of compression and shear waves of the samples show the same trends as the density. For the rocks of prehnitepumpellyite facies $V_{\mathrm{PR}}=6.32 \pm 0.39 \mathrm{~km} / \mathrm{s}, V_{\mathrm{SR}}=3.51$ $\pm 0.16 \mathrm{~km} / \mathrm{s}$.

The density values calculated by the mineral composition for rocks of prehnite-pumpellyite facies vary within $2.89-3.01 \mathrm{~g} / \mathrm{cm}^{3}$. The average densities for the entire set of the samples of prehnite-pumpellyite facies are $2.96 \pm 0.05 \mathrm{~g} / \mathrm{cm}^{3}$. The calculated densities are higher than experimentally measured, since they ignore a porosity inherent in real samples. The calculated densities also show lesser data spread.

The limits of changes in the calculated values of compression wave velocity are $6.15-6.44 \mathrm{~km} / \mathrm{s}$ for the rocks of prehnite-pumpellyite facies. The range of shear wave calculated velocities is $3.28-3.49 \mathrm{~km} / \mathrm{s}$ and their average value for all kinds of samples of prehnite-pumpellyite facies is $3.40 \pm 0.08 \mathrm{~km} / \mathrm{s}$. The experimentally measured and calculated values of compression wave velocities do not differ within the data scatter. Some difference is observed in the values of shear wave velocity.

The obtained values of elastic anisotropy factors showed that the rock samples from the River Soukerjoki area are both weakly and strongly anisotropic. Elastic anisotropy factors calculated on the basis of the shear wave velocity vary within $A_{\mathrm{P}}=$ $3.5-19 \%$. The anisotropy factor $B_{\mathrm{S}}$ calculated by the shear wave velocity is in the range of 2.5 to $10.3 \%$.

\section{GREENSCHIST FACIES}

Eleven samples of the greenschist facies were collected in the areas of Lake Tulyavr and the River Shuoniyoki, Figure 1. Photos of thin sections of the greenschist facies samples (crossed Nicol prisms) are
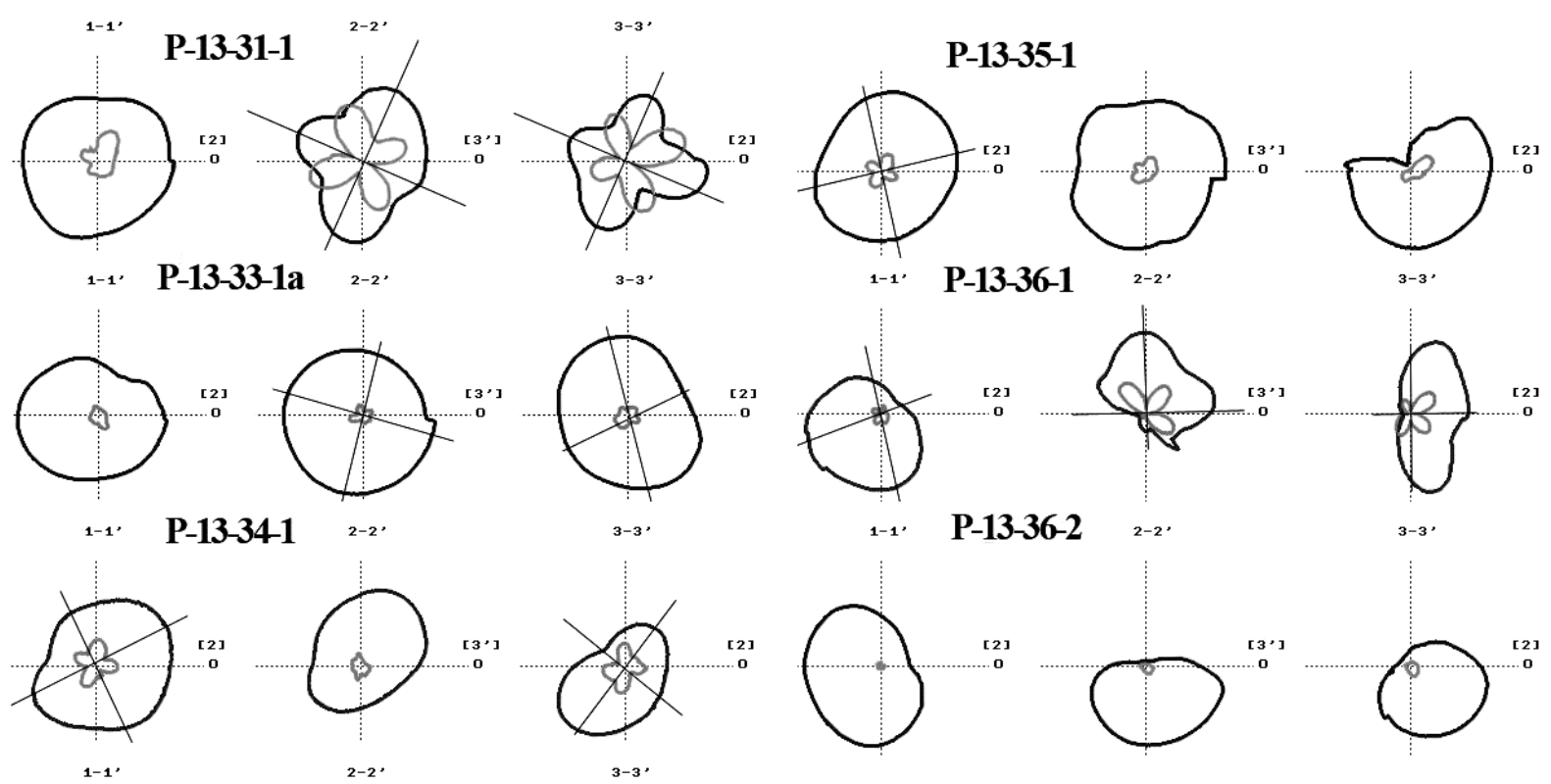

Fig. 5 Acoustopolarigrams of the samples of prehnite-pumpellyite facies. The dark line - parallel vectors, light crossed. 


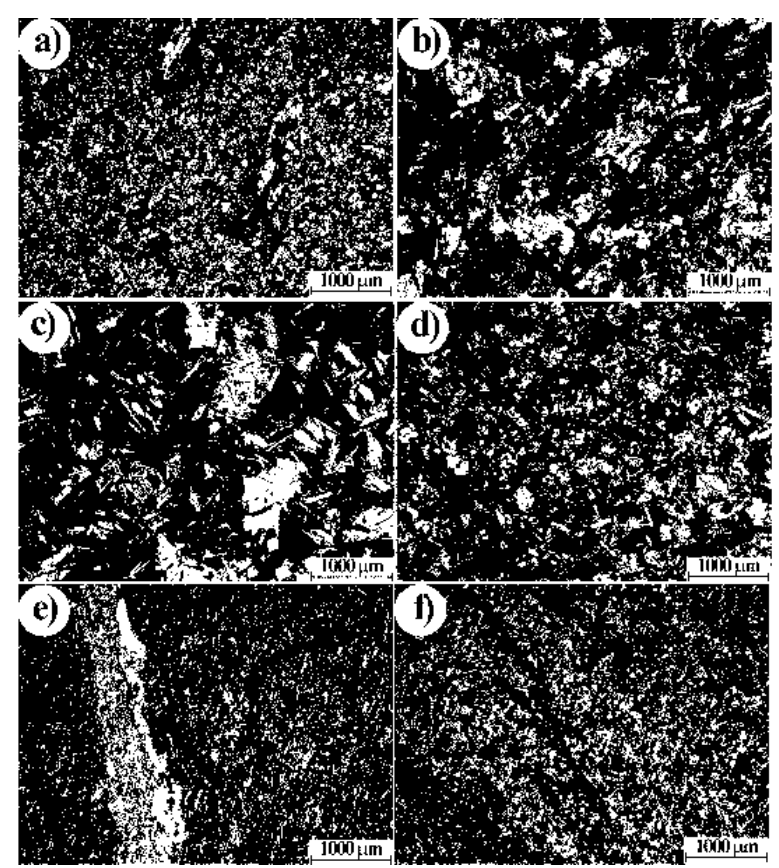

Fig. 6 Photo of some thin sections of the greenschist facies samples.

a) Metadiabase (P-13-11-2); b) metadiabase (P-1317-1a); c) gabbro (P-13-20-1); d) metagabbrodiabase (P-13-26-1a); f) metadiabase (P-13-39-1).

shown in Figure 6. The mineral composition and rock structure are given in Table 3 . The structure of metadiabases is granoblastic, nematogranoblastic, in places xenomorphic-granular, gabbroic and panidiomorphic. The main rock-forming minerals in the rock samples (in \%) are: plagioclase (24-49), chlorite (14-31), actinolite (1-23), sporadically observed quartz, potassic feldspar, epidote, biotite. Accessory minerals are apatite, titanite, carbonate, serpentine, ilmenite.

The VP diagrams of the greenschist facies rocks have rounded outlines. The projection orientation of the elastic symmetry elements was determined on the three faces of the cube by the VC acoustopolarigrams. The VC acoustopolarigrams are small. It is difficult to distinguish the direction of the medium symmetry elements in some of them. However the acoustopolarigrams of the samples P-13-39-1, P-1340-1, P-13-42-1 differ markedly from the others. The effect of linear acoustic anisotropic absorption is registered in them. For example, the photo of the metadiabase thin section (sample P-13-39-1) shows the presence of directive orientation of mineral grains. The VC diagrams for these samples allow one to distinguish clearly the direction of the elastic symmetry elements of the medium.

Analysis of petrophysical properties of greenschist facies (Table 4) shows that the average values of the sample density measured experimentally and calculated by the formula (6) for the whole variety are equal to $2.92 \pm 0.07 \mathrm{~g} / \mathrm{cm}^{3}$ and $2.96 \pm 0.05 \mathrm{~g} / \mathrm{cm}^{3}$, respectively. They hardly differ. The average velocities of compression and shear waves show the same trends as the density. The averages of experimentally measured and calculated velocities of compression and shear waves are, respectively, $V_{\mathrm{PR}}=$ $6.30 \pm 0.31 \mathrm{~km} / \mathrm{s}, V_{\mathrm{SR}}=3.51 \pm 0.17 \mathrm{~km} / \mathrm{s}, V_{\mathrm{PC}}=6.33$ $\pm 0.07 \mathrm{~km} / \mathrm{s}, V_{\mathrm{SC}}=3.44 \pm 0.06 \mathrm{~km} / \mathrm{s}$.

The obtained values of elastic anisotropy factors showed that the rock samples of greenschist facies are both weakly and strongly anisotropic. Anisotropy factors vary within $A_{\mathrm{P}}=2.0-14.5 \%, B_{\mathrm{S}}=3.0-9.5 \%$.

\section{EPIDOTE-AMPHIBOLITE FACIES}

Six samples of epidote-amphibolite facies were collected at the Valasjoki site, Figure. 1. Photos of the sample thin sections of epidote-amphibolite facies (crossed Nicol prisms) are shown in Figure 8. The amphibolite texture is nematogranoblastic, lepidogranoblastic, lepidonematogranoblastic and porphyry (Table 5). The main rock-forming minerals (in \%) are: plagioclase (40-51), hornblende (17-30), epidote (1021 ); accessories - quartz, biotite, ilmenite, orthoclase, apatite, serpentine, carbonate.

Analysis of the acoustopolarigrams (Fig. 9) for the samples of epidote-amphibolite facies indicates a more pronounced presence of elastic anisotropy of rocks and significant structural heterogeneity. This is evidenced by the petals outline of the VC diagrams and a distorted form of the VP diagrams as compared with theoretical predictions for a perfectly anisotropic medium (Gorbatsevich, 1999). The VC acoustopolarigrams for some samples (P-13-47-1, faces 2-2' and 3-3'; P-13-49-1a, faces 1-1' and 3-3') are minimal and occupy an area close to the origin of coordinates and it is impossible to determine the direction of the symmetry elements on them.

A comparison of acoustopolarigrams with the thin section photos allows one to notice that the irregular shape of the VP diagram for the sample R13-46-1 is explained by mineral inclusions in the rock matrix. Elastic anisotropy in the samples R-13-48-1 and $\mathrm{R}-13-50-1$ is caused by the directive orientation of the mineral grain forms with a relatively homogeneous texture.

According to the data from Table 6, the averages of the experimentally measured and calculated by the formula (6) densities for the entire variety are $3.01 \pm 0.08 \mathrm{~g} / \mathrm{cm}^{3}$ and $3.02 \pm 0.03 \mathrm{~g} / \mathrm{cm}^{3}$, respectively. They are virtually the same. However, experimentally measured and calculated average velocities of compression and shear waves differ markedly. The average experimentally measured and calculated velocities of compression and shear waves are, respectively, $V_{\mathrm{PR}}=5.13 \pm 0.78 \mathrm{~km} / \mathrm{s}, V_{\mathrm{SR}}=3.18 \pm 0.19$ $\mathrm{km} / \mathrm{s}, V_{\mathrm{PC}}=6.66 \pm 0.06 \mathrm{~km} / \mathrm{s}, V_{\mathrm{SC}}=3.69 \pm 0.02 \mathrm{~km} / \mathrm{s}$.

The obtained values of elastic anisotropy factors showed (see Table 6) that the rock samples of epidote-amphibolite facies are both weakly and strongly anisotropic. The anisotropy factors vary within $A_{\mathrm{P}}=1.6-7.6 \%, B_{\mathrm{S}}=2.4-17.4 \%$.

\section{AMPHIBOLITE FACIES}

Eight samples of mainly metagabbro and amphibolite were selected within the area of $\mathrm{Mt}$ Kuchin-tundra (Fig. 1). Photos of the samples thin sections (crossed Nicol prisms) are shown in 


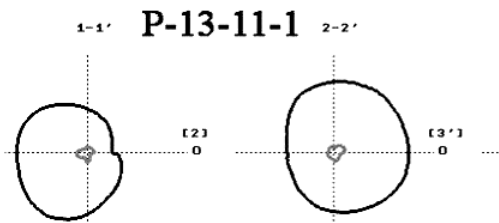

${ }_{1-1}$ P-13-11-2 2-2.
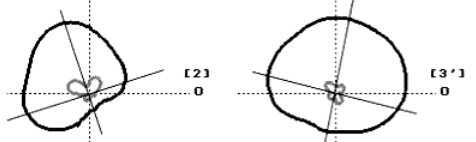

${ }^{1-1} \cdot$ P-13-17-1a ${ }^{2-2} \cdot$

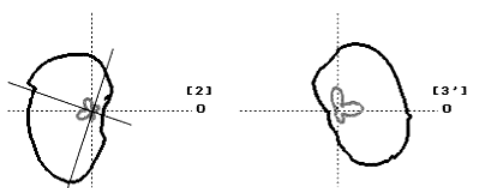

${ }_{1-1}, \mathrm{P}-13-20-1{ }_{2-2}$,

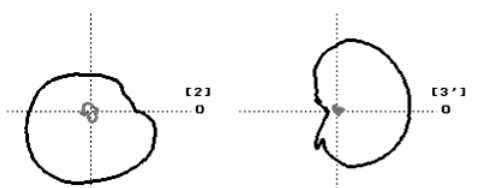

1-1. P-13-21-1

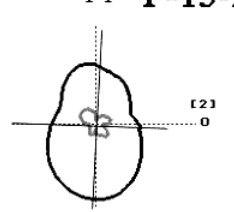

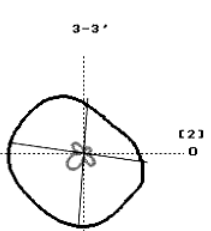

3-3.

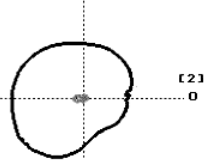

3-3,

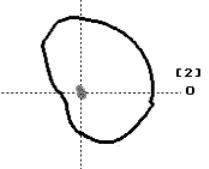

3-3.
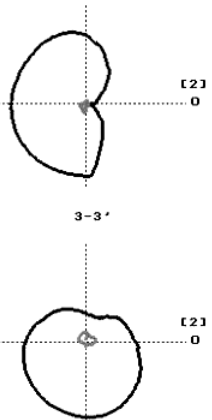

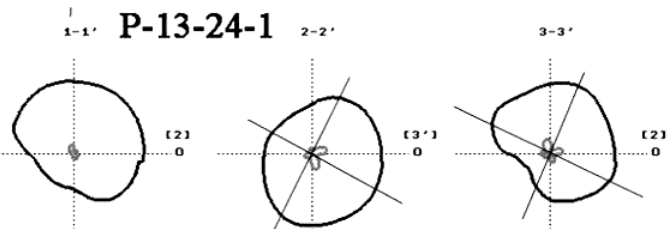

1-1. P-13-26-1 ${ }_{2-2}$.

3-3

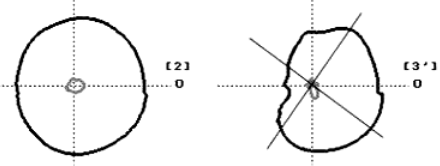

1-1. P-13-39-1 2-2,
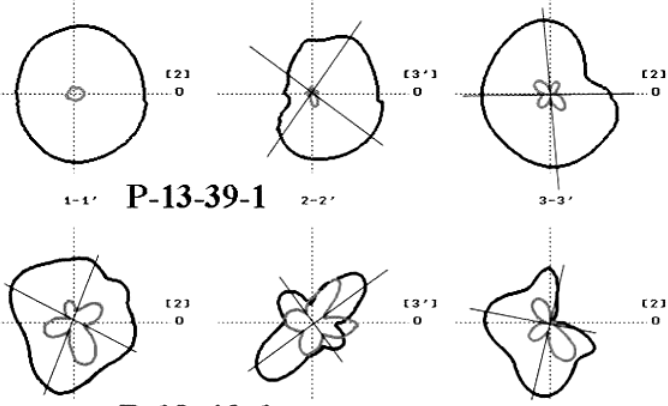

P-13-40-1

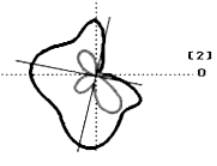

3-3.

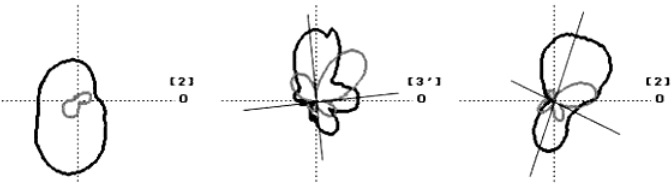

1-1, P-13-41-1 2-2.

3-3.
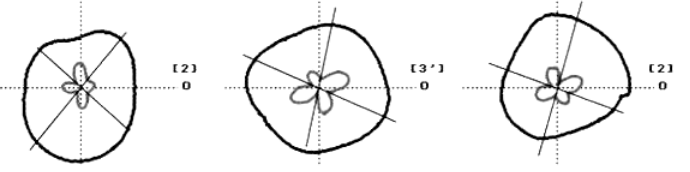

1-1. P-13-42-1 2-2.

3-3.
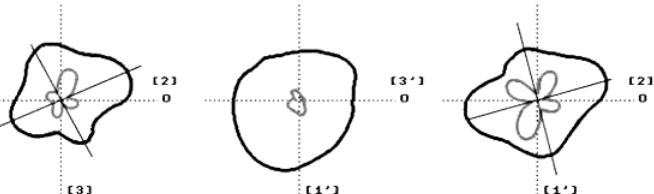

t1

(1.)

Fig. 7 Acoustopolarigrams of greenschist facies. Dark line - parallel vectors, light - crossed.
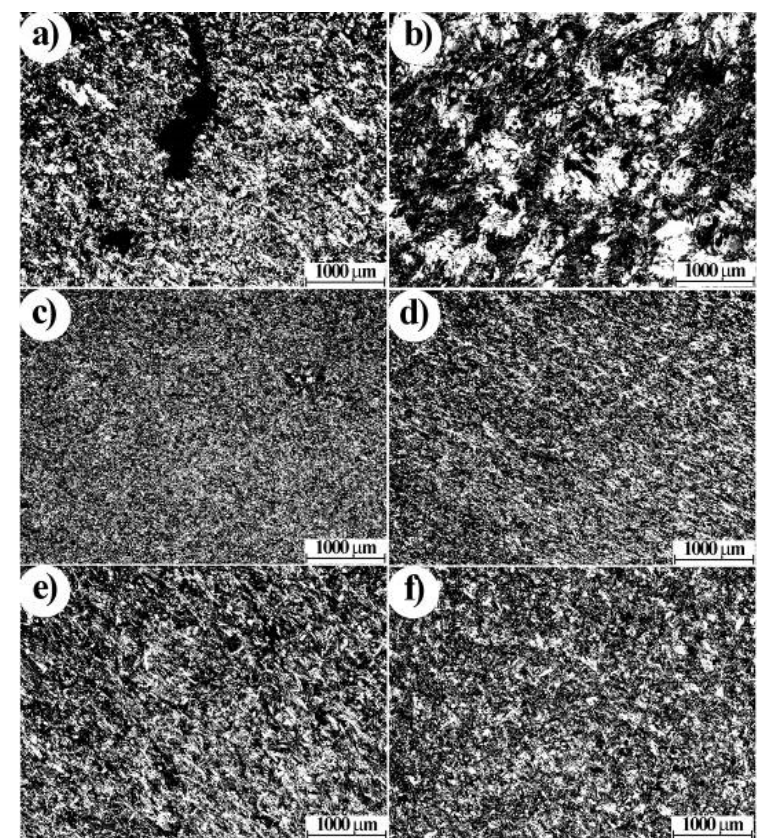

Fig. 8 Photo of thin sections of samples of epidoteamphibolite facies (amphibolites):

a) P-13-46-1; b) P-13-47-1; c) P-13-48-1;

d) P-13-49-1a; e) P-13-50-1; f) P-13-51-1.
Figure 10. Schistose amphibolite and metagabbro are represented by fine-grained, equigranular rocks of granonematoblastic, nematoblastic, relict gabbro, poikilitic texture. The mineral composition and the rock texture are shown in Table 7.

The main rock-forming minerals of amphibolite samples are amphibole 60-98\%, quartz and feldspar $20-34 \%$, ore minerals up to $5 \%$. The main minerals of metagabbro samples are clinopyroxene and plagioclase. Their maximum content in the samples is 30-35\%. Newly formed minerals (amphibole, biotite and carbonate) account for 25-30\%. Titanite constitutes up to $5 \%$, ore minerals are present as accessories $(<1 \%)$.

The projections orientation of elastic symmetry elements was determined on the three faces of the cubic samples using the acoustopolarigrams, Figure 11. Then the density and velocity of compression and shear waves were determined. The measurement results are shown in Table 8 . The rock samples of amphibolite facies show a clear dissimilarity in the acoustopolarigram shape from that of the rocks of prehnite-pumpellyite, greenschist and epidote- 


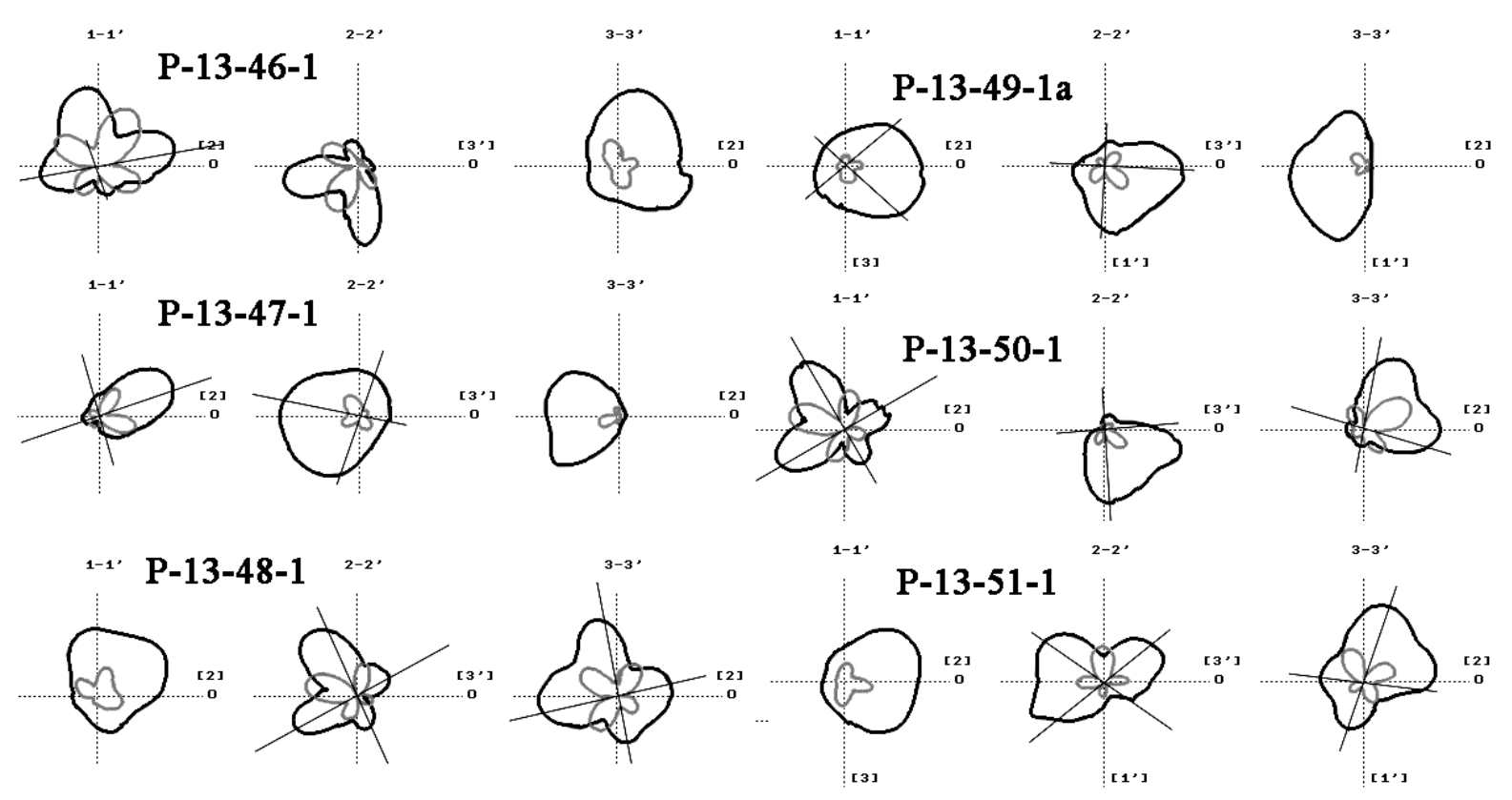

Fig. 9 Examples of acoustopolarigrams of epidote-amphibolite facies. Dark line - parallel vectors, light crossed.

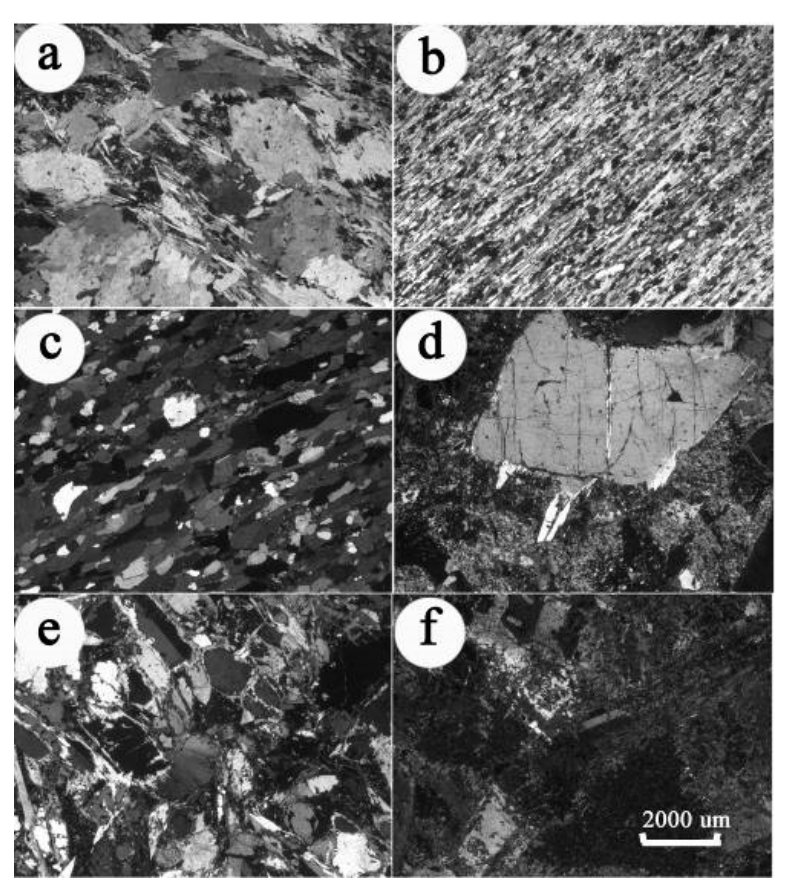

Fig. 10 Photo of the thin sections of the amphibolite facies samples. Amphibolites:

a) v-1731; b) v-1732; c) v-1734. Metagabbros: d) v-1741-8; e) v-1741-9; f) v-1741-11.

amphibolite facies. This distinction is observed both at parallel and crossed polarization vectors (Fig. 11). The acoustopolarigrams show that elastic anisotropy of the amphibolite facies is expressed more clearly. Hence it follows that these rocks have experienced a more profound degree of metamorphism. Rather small sizes of the petals of the VC diagram obtained for the samples v-1731, v-1732, v-1737-1 and v-1741-9 in the direction 1-1' allow one to conclude that the medium symmetry is close to transversely isotropic. It means that in the massif in the place of location of the samples the long acting unidirectional palaeostress state prevailed.

Some samples show a significant manifestation of the effect of linear acoustic anisotropic absorption. For example, the LAAA is expressed in the sample v1732 (face 2-2'). In the samples v-1734 (face 2-2' and 3-3') and v-1741-9 (face 2-2' and 3-3') this manifestation is significantly higher. This is an indication that there is a strict orientation of elongated in one direction forms of mineral grains in the rocks. Photos of thin sections confirm this (Fig. 10).

The averages for the entire variety of experimentally measured and calculated densities of the samples are equal to $3.04 \pm 0.12 \mathrm{~g} / \mathrm{cm}^{3}$ and 3.01 $\pm 0.17 \mathrm{~g} / \mathrm{cm}^{3}$, respectively (Table. 8). They do not differ virtually. However, experimentally measured and calculated average velocities of compression and shear waves differ markedly. Experimentally measured and calculated average velocities of compression and shear waves are, respectively, $V_{\mathrm{PR}}=$ $5.73 \pm 0.56 \mathrm{~km} / \mathrm{s}, V_{\mathrm{SR}}=3.34 \pm 0.35 \mathrm{~km} / \mathrm{s}, V_{\mathrm{PC}}=6.72$ $\pm 0.71 \mathrm{~km} / \mathrm{s}, V_{\mathrm{SC}}=3.79 \pm 0.29 \mathrm{~km} / \mathrm{s}$. The obtained factors of elastic anisotropy showed (see Table 8) that the rock samples of amphibolite facies are both weakly and strongly anisotropic. The anisotropy factors vary within $A_{\mathrm{P}}=0-18 \%, B_{\mathrm{S}}=5-35 \%$.

Technical constants are often calculated in mining. Elasticity modulus $E$, shear modulus $G$ and Poisson's ratio $v$ were calculated by the formulas (7-9) on the basis of the average density and velocity characteristics for all line of investigated rocks. Their average values for the surface and deep conditions are shown in Table 9. As derivatives of the density and velocity values these parameters reflect the behaviour of the data of density and velocity. 

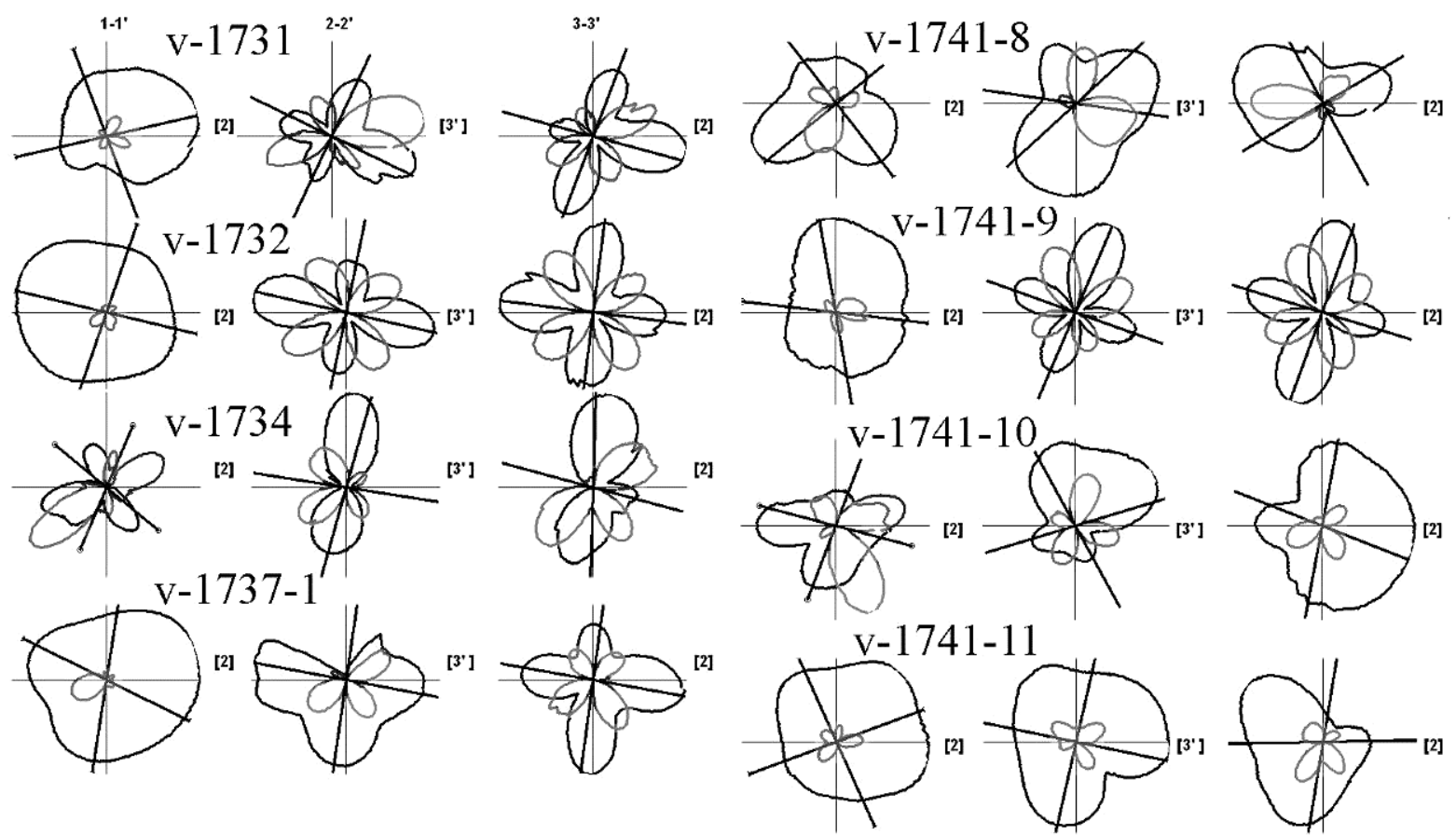

Fig. 11 Acoustopolarigrams of the samples of amphibolite facies. Dark line - vectors parallel, light - crossed.

\section{DISCUSSION}

In common, rocks of prehnite-pumpellyite facies - metadiabase, gabbro and gabbro-diabase are characterized by a relatively high homogeneity. They are characterized by fine-grained massive, more rarely schistose texture, which is reflected in the acoustopolarigram contours. The VP diagrams are close to circular, the $\mathrm{VC}$ diagrams are minimal and occupy an area close to the coordinate origin. The experimentally measured and calculated values of density and compression velocities do not differ within the data spread. This indicates high preservation of rocks, a relatively low impact of external factors (temperature and pressure) on their petrophysical properties during the past geological period.

In general, the elastic anisotropic properties of rocks of prehnite-pumpellyite and greenschist facies are virtually indistinguishable. Their experimentally measured and calculated densities can be regarded as the same within the scatter of unit data. The outlines of the VP and VC diagrams of both facies are very similar. A comparison of velocity data for prehnitepumpellyite and greenschist facies shows that the calculated data for the latter are slightly higher. At the same time, the average velocities measured for the surface conditions are practically identical. It is believed that despite the difference in the PTconditions of the compared facies, metamorphism did not change significantly the properties of these rocks.

Petrophysical properties of the samples of epidote-amphibolite facies are other than those of prehnite-pumpellyite and greenschist facies. The acoustopolarigrams of the samples of epidote- amphibolite facies show the presence of elastic anisotropy of rocks and significant heterogeneity of their texture. The VP acoustopolarigrams for the rocks of epidote-amphibolite facies are more complicated than those for prehnite-pumpellyite and greenschist facies. The directions that correspond to the orientation of elastic symmetry elements are distinguished on all acoustopolarigrams. Unlike rocks of prehnite-pumpellyite and greenschist facies the rock samples of this facies show a significant difference between the experimentally measured and calculated by the mineral composition velocities of compression and shear waves. This indicates greater metamorphic working of rocks of epidote-amphibolite facies.

The rocks of amphibolite facies metamorphism from the area of Mt Kuchin-tundra differ markedly from the rocks of the epidote-amphibolite, prehnitepumpellyite and greenschist facies. They show a significant difference between the experimentally measured and calculated by the mineral composition velocities of compression and shear waves. In general, rocks of amphibolite facies have undergone more substantial mineral rearrangement during metamorphic transformations than those of epidoteamphibolite facies. This resulted in a greater change of petrophysical properties of rocks from the $\mathrm{Mt}$ Kuchin-tundra area selected at the earth's surface.

It should be noted that the values of the density and velocity of compression waves (depth conditions) obtained for metadiabase, metagabbro, gabbro-diabase and amphibolite are in general close to those for the rocks described by Christensen and Mooney, (1995). The rocks selected on the earth's surface within the 
Pechenga structure showed a weak dependence of the density and velocity values on pressure and temperature, expressed only within the initial pressures (Kern et al., 2001). The determinations of the density and velocity of compression and shear waves, performed by the VSP method in the Proterozoic section of the Kola superdeep borehole (SG-3), where metamorphism passes from prehnitepumpellyite to amphibolite facies with depth, showed that these petrophysical properties do not depend on the depth (Kozlovsky, 1987; Digranes et al., 1996; Trčková et al., 2002). Therefore, in our opinion, for deep conditions it is fully justified to apply the method of calculating the average petrophysical properties by the mineral composition.

However, the complexity arises when evaluating elastic anisotropy of rocks. Rocks in the case of an inequilateral stressed state, which often occurs in the process of metamorphic transformations, acquire an oriented structure (Brace, 1960). In turn, oriented structures are the cause of the appearance of elastic anisotropy in rocks. Anisotropy in samples may be caused by preferred orientation of microcracks, crystal-lattice preferred orientation (LPO) and preferred orientation of grain shape (SPO) (Crampin, 1985; Smithson et al., 2000; Kern et al., 2001). In the samples selected on the Earth's surface all the listed reasons can manifest themselves. Therefore, instrumental methods should be used for estimating elastic anisotropy in metamorphosed rocks. The acoustopolariscopy method allows one to determine the orientation of the symmetry elements of an anisotropic body, without which it is impossible to measure the velocity characteristics of rocks correctly.

\section{CONCLUSION}

At the Matert Formation sites of the Pechenga synclinal structure petrophysical properties of rocks were studied: metadiabase, metagabbro, gabbrodiabase and amphibolite. The sites are represented by rocks metamorphosed within the prehnitepumpellyite, greenschist, epidote-amphibolite and amphibolite facies. It is shown how the petrophysical properties of rocks change when passing from the prehnite-pumpellyite to the amphibolite facies of metamorphism.

The study of physical properties of rocks of different facies of metamorphism showed that the density of rocks calculated for deep conditions increases from the prehnite-pumpellyite to the amphibolite facies. The lowest velocities of compression waves are observed in the rocks of the epidote-amphibolite and amphibolite facies (Earth's surface). This is most likely due to a profound structural rearrangement in the process of metamorphic transformations, the formation of secondary minerals, such as micas and amphiboles, which have low velocities in certain crystallographic directions, and weathering processes. The compression wave velocities, like the density, under deep conditions tend to increase from the prehnitepumpellyite to the amphibolite facies. The same situation is observed in changes in the shear wave velocities.

The derivatives of the density and velocity of compression and shear waves - the values of the elastic moduli retain the same tendencies in the transition from the prehnite-pumpellyite to the amphibolite facies. Low values of Poisson's ratio were recorded for the rocks of the epidote-amphibolite and amphibolite facies (Earth's surface) and for deep conditions a gradual decrease in the values of this ratio from the prehnite-pumpellyite to the amphibolite facies is observed.

A new quantitative method, - acoustopolariscopy was used in the studies. It can help distinguish rocks of different facies of metamorphism. It was revealed that the elastic characteristics of rocks are usually affected by factors encountered in various combinations and degrees of manifestation: inhomogeneities, elastic anisotropy, and the effect of linear acoustic anisotropic absorption. The acoustopolariscopy method significantly increases the amount of information obtained on a single rock sample.

Pechenga sulphide-nickel deposits are being developed by a number of mining enterprises. The data obtained (densities, velocity characteristics, moduli of elasticity and Poisson's ratios) can be used in the initial assessment of petrophysical properties of rocks in the course of geophysical studies and technical calculations in mining.

\section{ACKNOWLEDGMENTS}

The authors are grateful to V.R. Vetrin for describing the geological-geochemical features and metamorphism of the Pechenga structure rocks, V.F. Smolkin (V.I. Vernadsky Geological Museum, Moscow) for the provided samples for research and results of analytical studies and discussion of the results obtained, V.P. Petrov for useful consultations.

This work was supported by the Russian Foundation for Basic Research (grants No. 13-0500125-a, 16-05-00026-a).

\section{REFERENCES}

Aleksandrov, K.S. and Ryzhova, T.V.: 1961a, The elastic properties of crystals, Soy. Phys. Crystallogr., 6, 228252.

Allison, I.S. and Palmer, D.F.: 1980, Geology. The Science of a Changing Earth. McGraw-Hill Book Co.

Belikov, B.P., Aleksandrov, K.S. and Ryzhova, T.V.: 1970, Elastic properties of rock forming minerals and rocks. Moscow, Nauka, 276 pp, (in Russian).

Berckhemer, H., Rauen, A., Winter, H., Kern, H., Kontny, A., Lienert, M., Nover, G., Pohl, J., Popp, T., Schult, A., Zinke, J. and Soffel, H.C.: 1997, Petrophysical properties of the 9-km-deep crustal section at KTB. J. Geophys. Res., 102 (B8), 18337-18361.

DOI: $10.1029 / 96 J B 03396$ 
Berry, L.G., Mason, B. and Dietrich, R.V.: 1983, Mineralogy. W.H. Freeman and Company. San Francisco.

Brace, W.F.: 1960, Orientation of anisotropic minerals in a stress field: discussion. Mem. Geol. Soc. Amer. No. 79, 9-20.

Christensen, N.I. and Mooney, W.D.: 1995, Seismic velocity structure and composition of the continental crust: a global view. J. Geophys. Res., 100 (B6), 9761-9788. DOI: 10.1029/95JB00259

Crampin, S.: 1985, Evaluation of anisotropy by shear-wave splitting. Geophysics, 50 (1), 142-152. DOI: $10.1190 / 1.1441824$

Digranes, P., Kristoffersen, Y. and Karajev, N.: 1996, An analysis of shear waves observed in VSP data from the superdeep well at Kola, Russia. Geophys. J. Int., 126, 545-554. DOI: 10.1111/j.1365-246X.1996.tb05309.x

Glebovitsky, V.A.: 1973, Evolution aspects of metamorphic processes in mobile regions. Leningrad: Nauka, 127 $\mathrm{pp}$, (in Russian).

Golovataya, O.S., Gorbatsevich, F.F., Kern, H. and Popp, T.: 2006, Properties of some rocks from the section of the Kola ultradeep borehole as a function of the P-T parameters. Izvestiya, Physics of the Solid Earth, 42 (11), 865-876. DOI: 10.1134/S1069351306110012

Gorbatsevich, F.: 2009, Acoustopolariscopy of Minerals and Rocks. VDM Verlag, Saabrucken.

Gorbatsevich, F.F.: 2003, Decompaction mechanism of deep crystalline rocks under stress relief. Tectonophysics, $370,1-4,121-128$

DOI: $10.1016 / \mathrm{S} 0040-1951(03) 00181-1$

Gorbatsevich, F.F.: 1999, Acoustic polarization method for determining elastic symmetry and constants of anisotropy in solid media. Ultrasonics, 37, 309-319. DOI: $10.1016 / \mathrm{S} 0041-624 \mathrm{X}(98) 00064-\mathrm{X}$

Gorbatsevich, F.F., Smirnov, Yu.P. and Medvedev, R.V.: 1997, Elastic-anisotropic properties of the core from the cross-section of the Kola super deep (SD-3): Measurements by means of acoustopolariscopy. Scientific Drilling, 6, 103-111.

Handbook (cadastre) of physical properties of rocks: 1975, Ed. Protod'yakonov, M.M. Moscow, Nedra, 279 pp., (in Russian).

Hanski, E.J., Huhma, H., Smol'kin, V.F. and Vaasjoki, M.: 1990, The age of ferropicritic volkanites and comagmatic Ni-bearing intrusions at Pechenga, Kola Peninsula, U.S.S.R. Geol. Soc. Finl. Bull, 62, 123 133.

Kern, H., Popp, T., Gorbatsevich, F., Zharikov, A., Lobanov, K.V. and Smirnov, Yu.P.: 2001, Pressure and temperature dependence of $\mathrm{Vp}$ and $\mathrm{Vs}$ in rocks from the superdeep well and from surface analogues at Kola and the nature of velocity anisotropy. Tectonophysics, 338, 113-134.

DOI: 10.1016/S0040-1951(01)00128-7
Kern, H. and Schmidt, R.: 1990, Physical properties of KTB core samples at simulated in situ conditions. Scientific Drilling (1), 217-223.

Kern, H., Mengel, K., Strauss, K.W., Ivankina, T.I., Nikitin, A.N. and Kukkonen, I.T.: 2009, Elastic wave velocities, chemistry and modal mineralogy of crustal rocks sampled by the Outokumpu scientific drill hole: Evidence from lab measurements and modeling. Physics of the Earth and Planetary Interiors, 175, 151166. DOI: 10.1016/j.pepi.2009.03.009

Kovalevskiy, M.V.: 2009, The automated software and hardware complex Acoustpol: Tutorial. Apatity, "K \& M" Publ., 54 pp, (in Russian).

Kozlowski, Ye.A., ed.: 1987, The super-deep well of Kola Peninsula, Springer, Berlin, Heidelberg, New York, Tokyo.

Kretz, R.: 1983, Symbols for rock-forming minerals. Amer. Mineral., 68, 277-279.

Patent No. 1281993, USSR: 1987, MKI G01N 29/04. Acoustopolariscope for measuring elasticity of solid media samples. Byull. Izobret., (1), (in Russian).

Patent No. 785737, USSR: 1980, MKI G01N 29/04. Transducer of shear ultrasonic waves. Byull. Izobret., (45), (in Russian).

Petrov, V.P.: 1999, Metamorphism of the Early Proterozoic of the Baltic Shield, Apatity. KSC RAS Publ., 325 pp, (in Russian).

Rudnick, R.L. and Fountain, D.M.: 1995, Nature and composition of the continental crust: a lower crustal perspective, Rev. Geophysics, 33, 267-309. DOI: 10.1029/95RG01302

Shurkliff, W.A.: 1962. Polarized light. Harvard University Press.

Smithson, S.B., Wenzel, F., Ganchin, Y.V., and Morozov, I. B.: 2000, Seismic results at Kola and KTB deep scientific boreholes: velocities, reflections, fluids, and crustal composition. Tectonophysics, 329, 301-317. DOI: 10.1016/S0040-1951(00)00200-6

Smol'kin, V.F.: 1992, Komatiitic and picritic magmatism of the Early Precambrian of the Baltic Shield. SPb: Nauka, 272 pp, (in Russian).

Trčková, J., Živor, R., Kazansky, V.I., Lobanov, K.V., Zharikov, A.V. and Smirnov, Y.P.: 2002, Comparison of elastic properties of the Kola Superdeep borehole core samples and their surface analogues obtained by static and dynamic measurements. Acta Montana IRSM AS CR Series A, 21 (125), 27-54.

Zagorodny, V.G., Mirskaya, D.D. and Suslova, S.N.: 1964, Geological structure of the Pechenga sedimentaryvolcanogenic group. Moscow-Leningrad, Nauka. 207 $\mathrm{pp}$, (in Russian). 